\title{
EMISSION FROM PAIR-INSTABILITY SUPERNOVAE WITH ROTATION
}

\author{
E. Chatzopoulos ${ }^{1,7}$, Daniel R. van Rossum ${ }^{1}$, Wheeler J. Craig ${ }^{2}$, Daniel J. Whalen ${ }^{3}$, \\ JOSEPH SMIDT ${ }^{4}$, AND BRANDON WIGGINS ${ }^{5,6}$ \\ ${ }^{1}$ Department of Astronomy \& Astrophysics, Flash Center for Computational Science, University of Chicago, \\ Chicago, IL 60637, USA; manolis@ flash.uchicago.edu \\ ${ }^{2}$ Department of Astronomy, University of Texas at Austin, Austin, TX 78712, USA \\ ${ }^{3}$ Universität Heidelberg, Zentrum für Astronomie, Institut für Theoretische Astrophysik, \\ Albert-Ueberle-Strasse. 2, D-69120 Heidelberg, Germany \\ ${ }^{4}$ T-2, Los Alamos National Laboratory, Los Alamos, NM 87545, USA \\ ${ }^{5}$ CCS-2, Los Alamos National Laboratory, Los Alamos, NM 87545, USA \\ ${ }^{6}$ Department of Physics and Astronomy, Brigham Young University, Provo, UT 84602, USA \\ Received 2014 September 30; accepted 2014 November 4; published 2015 January 12
}

\begin{abstract}
Pair-instability supernovae (PISNe) have been suggested as candidates for some superluminous supernovae, such as SN 2007bi, and as one of the dominant types of explosion occurring in the early universe from massive, zerometallicity Population III stars. The progenitors of such events can be rapidly rotating, therefore exhibiting different evolutionary properties due to the effects of rotationally induced mixing and mass-loss. Proper identification of such events requires rigorous radiation hydrodynamics and radiative transfer calculations that capture not only the behavior of the light curve but also the spectral evolution of these events. We present radiation hydrodynamics and radiation transport calculations for $90-300 M_{\odot}$ rotating PISNe covering both the shock breakout and late light curve phases. We also investigate cases of different initial metallicity and rotation rate to determine the impact of these parameters on the detailed spectral characteristics of these events. In agreement with recent results on non-rotating $\mathrm{PISNe}$, we find that for a range of progenitor masses and rotation rates these events have intrinsically red colors in contradiction with observations of superluminous supernovae. The spectroscopic properties of rotating PISNe are similar to those of non-rotating events with stripped hydrogen and helium envelopes. We find that the progenitor metallicity and rotation rate properties are erased after the explosion and cannot be identified in the resulting model spectra. It is the combined effects of pre-supernova mass-loss and the basic properties of the supernova ejecta such as mass, temperature, and velocity that have the most direct impact in the model spectra of PISNe.
\end{abstract}

Key words: radiative transfer - stars: massive - stars: rotation - supernovae: general supernovae: individual (pair-instability)

\section{INTRODUCTION}

The importance of understanding the supernova (SN) explosions of very massive stars $\left(M_{\text {ZAMS }}>50 M_{\odot}\right)$ is pertinent in both low and high redshift. Recent developments in transient astrophysics led to the discovery of a new class of relatively nearby $(z \sim 0-1.2)$ explosions with peak luminosities $\sim 10-100$ greater than those of all types of core-collapse supernova (CCSN) events and with a striking degree of diversity in their emission properties, the superluminous supernova (SLSN) events (Quimby et al. 2007; Smith et al. 2007, see also Gal-Yam 2012 for a review and more references).

Although the extreme luminosities of the majority of the observed SLSNe are attributable to other processes, such as strong SN ejecta-circumstellar matter (CSM) interaction (Smith \& McCray 2007; Chatzopoulos et al. 2011; Chatzopoulos \& Wheeler 2012b; Moriya \& Tominaga 2012; Moriya et al. 2013; Chatzopoulos et al. 2013b; Benetti et al. 2014; Nicholl et al. 2014) or efficiently thermalized proto-magnetar spin-down radiation (Kasen \& Bildsten 2010; Woosley 2010; Dessart et al. 2012; Lunnan et al. 2014; McCrum et al. 2014), there are still a few SLSN light curves (LCs) that seem to be well fit by explosions of very massive progenitor stars $\left(M_{\text {ZAMS }}>130 M_{\odot}\right)$ powered by the radioactive decay of large amounts of ${ }^{56} \mathrm{Ni}$ $\left(M_{\mathrm{Ni}}>1-10 M_{\odot}\right.$, Heger \& Woosley 2002; Woosley et al. 2007)

\footnotetext{
7 Enrico Fermi Fellow.
}

with the most well-studied case being SN 2007bi (Gal-Yam et al. 2009; Gal-Yam 2012; Kasen et al. 2011; Kozyreva et al. 2014a, 2014b).

These pair-instability supernovae (PISNe; Rakavy \& Shaviv 1967; Barkat et al. 1967; Rakavy et al. 1967; Ober et al. 1983; Heger \& Woosley 2002; Chatzopoulos \& Wheeler 2012a; Chatzopoulos et al. 2013a; Yusof et al. 2013; Chen et al. 2014a, $2014 b$ ) occur when the helium cores of very massive stars enter a regime of high temperature $\left(\gtrsim 10^{9} \mathrm{~K}\right)$ and relatively low density $\left(\sim 10^{3}-10^{6} \mathrm{~g} \mathrm{~cm}^{-3}\right)$ allowing rapid production of electron-positron $\left(\mathrm{e}^{-}-\mathrm{e}^{+}\right)$pairs $\left(M_{\text {ZAMS }} \simeq 130-260 M_{\odot}\right.$, $M_{\mathrm{CO}} \simeq 60-140 M_{\odot}$ for non-rotating progenitors at ZAMS, where $M_{\mathrm{CO}}$ is the mass of the He core; Heger \& Woosley 2002; Woosley et al. 2007). Note that we use the "CO" notation since $75 \%$ of the He core is, in reality, $\mathrm{C}$ and $\mathrm{O}$. The rapid electronpositron pair production leads to the reduction of radiation pressure in the core and the decrease of the adiabatic index, $\Gamma_{\text {ad }}$, below $4 / 3$ resulting in core contraction and, consequently, explosive nuclear burning and the production of a strong SN shock that totally disrupts the star.

A few very massive stars (VMS) that seem to fit the criteria to be PISN progenitors have been discovered (Crowther et al. 2010). At near to solar metallicities, models predict that the effects of radiatively driven mass-loss are so strong that they can drive the mass of the star below the range required for PISNe (Langer et al. 2007; but see Kozyreva et al. 2014a for an alternative view). In fact, the measured metallicities of most 
SLSN hosts are always larger than $\sim 0.1 Z_{\odot}$ (Stoll et al. 2013; Lunnan et al. 2014).

The prevalence of PISNe is therefore expected to be higher in very low- to zero-metallicity environments characteristic of the conditions in the early universe. The Population III stars are found to be quite massive in primordial star formation simulations, well in the PISN regime (Abel et al. 1998; Bromm et al. 2002; Bromm \& Larson 2004; Stacy et al. 2012, 2013). Pending the deployment of future missions such as the James Webb Space Telescope (JWST) and the Wide Field Infrared Survey Telescope (WFIRST), it has been broadly proposed to look for the primordial PISNe explosions resulting from the first stars (Scannapieco et al. 2005; Bromm et al. 2009; Joggerst \& Whalen 2011; Pan et al. 2012; Hummel et al. 2012; Whalen et al. 2013a, 2013b; Whalen 2013; de Souza et al. 2014).

Since PISNe can be linked to both SLSNe and primordial Pop III SNe, it is critical to understand their radiative properties across the relevant parameter space. So far the corresponding efforts have focused on non-rotating PISN progenitors on the high end of the mass range. Gray flux limited diffusion (FLD) radiation hydrodynamics model LCs have been presented in the context of the detectability of primordial PISNe (Scannapieco et al. 2005; Whalen et al. 2013a, 2013b) but also to compare to the observed LCs of some SLSNe such as SN 2006gy (Woosley et al. 2007) and SN 2007bi, and PTF10nmn (Gal-Yam et al. 2009; Kasen et al. 2011; Kozyreva et al. 2014a, 2014b).

A rigorous, self-consistent comparison with observed SN spectra requires accurate modeling of the observed spectral evolution and not just the bolometric and broadband LCs. Dessart et al. (2013) demonstrated this fact by non-local thermal equilibrium (non-LTE) radiative transfer calculations yielding model non-rotating PISN spectra as a function of time with the CMFGEN code (Hillier \& Dessart 2012). They found that PISNe are intrinsically too red in color as compared to SLSN events like SN 2007bi, and that their spectral evolution does not match that of any observed SN so far.

Recently, it has been shown that the inclusion of the effects of rotation in the evolution of PISN progenitors changes some key characteristics of these events (Chatzopoulos \& Wheeler 2012a, 2012b; Yoon et al. 2012; Chatzopoulos et al. 2013a). More specifically, rotationally induced mixing can shift the mass limit to encounter PISN to lower values $\left(85 M_{\odot}<M_{\text {ZAMS }}<190 M_{\odot}\right.$ at rotation rates $50 \%$ that of the critical Keplerian value). In addition, the combined effects of mixing and rotationally induced mass-loss often lead to bare $\mathrm{CO}$ cores (stripped totally of both $\mathrm{H}$ and $\mathrm{He}$ ) prior to explosion and to differences in mixing, ${ }^{56} \mathrm{Ni}$ yields, and energetics, therefore changing the interplay between energy input and SN ejecta opacity. This alone may have an effect on the radiative properties of these events as compared to non-rotating PISNe.

Rapid stellar rotation has been observed for some massive nearby stars (Dufton et al. 2011) and it is also found in simulations of primordial massive star formation (Greif et al. 2011; Stacy et al. 2013). To further complement the parameter space relevant to PISN explosions, in this work we present radiation hydrodynamics and LTE radiative transfer calculations of PISNe resulting from rotating progenitors and compare our results to the non-rotating case and to some observed $\mathrm{SNe}$ and the PISN candidate SN 2007bi.

The paper is organized as follows. In Section 2 we present the 1D stellar evolution and hydrodynamics simulations of rotating PISN progenitors up to a time prior to shock breakout (SBO).
In Section 3 we discuss the SBO emission using results from radiation hydrodynamics simulations. In Section 4 we present LTE LCs and spectra of rotating PISNe and compare with results from non-rotating models (Kasen et al. 2011; Dessart et al. 2013) as well as observations of SLSNe like SN 2007bi and other events. Last, in Section 5 we summarize and discuss our conclusions.

\section{STELLAR EVOLUTION AND HYDRODYNAMICS SIMULATIONS}

In order to obtain time-dependent spectra and final LCs for rotating PISNe, we followed a multi-step process. First we evolved a grid of progenitors from the zero-age main sequence (ZAMS) up to the point when the dynamical instability is encountered using the stellar evolution code Modules for Experiments in Stellar Astrophysics (MESA; Paxton et al. 2011, 2013). At this point, the structure of the star was becoming dynamical and the semi-hydrostatic stellar evolution code reached its limitations. For this reason, we conservatively mapped the MESA models to the 1D Adaptive Mesh Refinement (AMR) grid of the multi-physics hydrodynamics code FLASH (Fryxell et al. 2000; Dubey et al. 2009) and followed the evolution of the explosion up to the epoch before SN SBO. Since we needed to capture the full radiation hydrodynamics nature of SN SBO involving the separation of matter and radiation temperatures, the next step involved re-mapping of the blast profiles to the radiation hydrodynamics code $R A G E$ (Gittings et al. 2008; Frey et al. 2013). The RAGE profiles were post-processed by the Los Alamos National Lab (LANL) SPECTRUM (Frey et al. 2013) code yielding SBO LCs and spectra. Finally, once the SN ejecta were expanding homologously the post-SBO profiles were mapped in the Lagrangian grid of the radiative transfer code PHOENIX (Hauschildt 1992; Hauschildt \& Baron 1999, 2004; van Rossum 2012a) that provided us with time-dependent spectra and LCs of rotating PISNe. We only calculated SBO LCs for 10 zero-metallicity rotating models because PISN models of higher metallicity and zero rotation have already been investigated (Kasen et al. 2011; Dessart et al. 2013).

\subsection{MESA Pre-supernova Evolution}

We modeled the evolution of 10 PISN progenitor stars with zero initial ZAMS metallicity, rotation at $50 \%$ that of the critical Keplerian value at the equator and in the mass range $90-140 M_{\odot}$ using MESA version 5596. This mass range was motivated by the finding of Chatzopoulos \& Wheeler (2012a) and Yoon et al. (2012) that full-fledged zero-metallicity PISNe that rotate with $\Omega / \Omega_{\mathrm{c}, \text { ZAMS }}=0.5$ occur for ZAMS masses $>85 M_{\odot}$. In the above expression, $\Omega$ and $\Omega_{\mathrm{c}, \mathrm{ZAMS}}$ are the angular velocity at the equator and the critical value of angular velocity at ZAMS, respectively.

The zero-metallicity models were considered because of their relevance to primordial star formation and the proposals to look for the PISN explosions from the first massive stars (Scannapieco et al. 2005; Whalen et al. 2013a, 2013b). For that same reason, we adopted an initial rotation rate of $\Omega / \Omega_{c, \text { ZAMS }}=$ 0.5 that seems to be a characteristic value found in early universe star formation simulations (Greif et al. 2011; Stacy et al. 2013).

In order to properly assess the parameter space, but also to study models relevant to nearby recently discovered PISN candidates such as SN 2007bi, we calculated two rotating PISN models at higher metallicities $\left(260 M_{\odot}\right.$ at $0.05 Z_{\odot}$ and $300 M_{\odot}$ at $0.1 Z_{\odot}$ at the ZAMS both with $\Omega / \Omega_{\mathrm{c}, \mathrm{ZAMS}}=0.5$ ). 
Table 1

Properties of PISN Progenitor Models

\begin{tabular}{lcrcccrr}
\hline \hline $\begin{array}{l}M_{\text {ZAMS }} \\
\left(M_{\odot}\right)\end{array}$ & $\begin{array}{c}Z_{\text {ZAMS }} \\
\left(Z_{\odot}\right)\end{array}$ & $\begin{array}{c}R_{\mathrm{f}} \\
\left(10^{10} \mathrm{~cm}\right)\end{array}$ & $\begin{array}{c}M_{\mathrm{f}} \\
\left(M_{\odot}\right)\end{array}$ & $\begin{array}{c}M_{\mathrm{CO}} \mathrm{b} \\
\left(M_{\odot}\right)\end{array}$ & $\begin{array}{c}M_{\mathrm{Ni}} \\
\left(M_{\odot}\right)\end{array}$ & $\begin{array}{c}E_{\mathrm{SN}}-E_{\mathrm{b}, \mathrm{f}} \mathrm{c} \\
\left(10^{52} \mathrm{erg}\right)\end{array}$ & $\begin{array}{c}M_{\mathrm{bol}, \mathrm{peak}} \\
(\mathrm{mag})\end{array}$ \\
\hline 90 & 0.0 & 3.925 & 60.51 & 59.31 & 0.138 & 0.98 & -16.08 \\
95 & 0.0 & 5.598 & 68.97 & 63.48 & 0.327 & 1.07 & -16.93 \\
105 & 0.0 & 4.730 & 71.12 & 69.10 & 0.853 & 2.84 & -17.96 \\
110 & 0.0 & 5.707 & 74.93 & 70.35 & 1.141 & 3.94 & -18.15 \\
115 & 0.0 & 8.266 & 76.73 & 70.48 & 1.070 & 3.12 & -18.05 \\
120 & 0.0 & 6.565 & 79.03 & 72.57 & 1.565 & 4.28 & -18.52 \\
125 & 0.0 & 8.034 & 83.60 & 76.80 & 3.255 & 5.04 & -19.30 \\
130 & 0.0 & 10.616 & 85.64 & 77.69 & 3.870 & 5.23 & -19.47 \\
135 & 0.0 & 18.324 & 90.15 & 79.76 & 4.521 & 6.16 & -19.66 \\
140 & 0.0 & 7.649 & 91.30 & 83.71 & 7.297 & 7.95 & -20.24 \\
$140 \mathrm{pF}$ & 0.1 & 7.649 & 91.30 & 85.60 & 6.453 & 7.62 & -20.24 \\
$140 \mathrm{pP}$ & 0.1 & 7.649 & 91.30 & 83.71 & 7.297 & 7.95 & -20.24 \\
$140 \mathrm{no}-\mathrm{ST}$ & 0.0 & 22.341 & 91.82 & 85.17 & 2.489 & 3.08 & -19.20 \\
$200^{\mathrm{a}}$ & 0.0 & 3772.167 & 111.28 & 82.48 & 0.165 & 1.55 & -16.00 \\
$260^{\mathrm{a}}$ & 0.0 & 1398.403 & 139.43 & 95.11 & 9.858 & 4.94 & -20.50 \\
260 & 0.05 & 7.299 & 116.85 & 112.02 & 22.22 & 6.64 & -21.50 \\
300 & 0.1 & 9.322 & 111.02 & 111.02 & 12.70 & 4.78 & -20.92 \\
\hline
\end{tabular}

Notes.

a These models were evolved without rotation.

${ }^{\mathrm{b}} M_{\mathrm{CO}}$ is the mass of the carbon-oxygen core defined as the total mass within the radius where $X_{\mathrm{C}}+X_{\mathrm{O}}>0.5$.

${ }^{c}$ The nuclear energy released by the $\mathrm{SN}$ explosion, $E_{\mathrm{SN}}$, minus the pre-SN binding energy of the progenitor, $E_{\mathrm{b}, \mathrm{f}}$.

These large ZAMS masses are characteristic of the most massive stars observed (Crowther et al. 2010). Very large ZAMS mass is required in order to counter the strong effects of radiatively driven mass-loss at these high metallicities allowing the formation of final cores with masses in the PISN regime. To determine whether the presence of metals in otherwise identical PISNe can be detected in model spectra, we artificially added metal content in the $140 M_{\odot}$ model assuming $Z=0.1$ $Z_{\odot}$ prior to mapping to $P H O E N I X$ and re-normalized all the abundances ("pP" model). In addition, to determine whether the presence of the same metal content in the $140 M_{\odot}$ model has an effect on the explosive PISN nucleosynthesis we also added the same metal content $\left(0.1 Z_{\odot}\right)$ prior to mapping to $F L A S H$ (" $\mathrm{pF}$ " model). Although the models with artificially added metallicity ("pP" and " $\mathrm{pF}$ ") are not entirely self-consistent since the presence of metals will itself change the pre-SN evolution, we use these models to gain intuition on the magnitude of the effect.

Finally, to probe spectroscopic differences that might arise due to varying degrees of pre-PISN rotation rate, we calculated two zero-metallicity non-rotating models (with $M_{\text {ZAMS }}=200$ and $260 M_{\odot}$ ) and a $140 M_{\odot}$ model with $\Omega / \Omega_{\mathrm{c}, \mathrm{ZAMS}}=0.5$ but without the effects of mixing and angular momentum transport due to the magnetic fields by turning off the Spruit-Taylor (ST) mechanism (140 $M_{\odot}$ "no-ST" model). The absence of magnetic viscosity in this model led to a much faster rotating pre-PISN core $\left(\Omega / \Omega_{\mathrm{c}} \simeq 0.3\right)$. As a result we calculated a total of four $140 M_{\odot}$ models spanning the rotation and metallicity parameter space: the original model with the effects of ST included, one without the effects of ST and two with artificially added metallicity $\left(0.1 Z_{\odot}\right)$ prior to doing the PISN nucleosynthesis in FLASH and prior to mapping to PHOENIX to calculate the radiative transfer properties. For details on the MESA parameters used (equation of state, nuclear reaction network, resolution, treatment of mass-loss and rotation) refer to Chatzopoulos \& Wheeler (2012a). The basic properties of the PISN models we examine in this work are listed in Table 1.
The effects of rotation and magnetic fields via the ST mechanism (Spruit 1999, 2002) on the evolution of massive stars have been investigated thoroughly in the past with the use of different stellar evolution codes (Heger et al. 2000; Yoon \& Langer 2005; Brott et al. 2011a, 2011b; Ekström et al. 2008, 2012; Chatzopoulos \& Wheeler 2012b; Yoon et al. 2012), also see (Maeder \& Meynet 2011) for a review. Enhanced chemical mixing, primarily due to meridional circulation and the ST mechanism, can lead to the formation of more massive $\mathrm{CO}$ cores for a lower ZAMS mass than required in the case of zero rotation. Furthermore, diffusion of angular momentum can lead to additional, mechanically induced mass-loss (Heger et al. 2000) that in turn can, under some circumstances, result in the stripping of both the $\mathrm{H}$ and $\mathrm{He}$ envelopes from the $\mathrm{SN}$ progenitor star leaving a bare $\mathrm{CO}$ core remnant.

This is the case for most of the models we consider in this work. In particular, we stopped the MESA calculations when a significant portion of the pre-PISN CO cores was in the pair-instability regime of $\Gamma_{\mathrm{ad}}<4 / 3$ and the model was becoming dynamical. In general this was the phase of core carbon exhaustion, in agreement with (Dessart et al. 2013). Figure 1 shows the temperature-density $(\rho-T)$ structure of the $140 M_{\odot}$ model when the $M E S A$ run was interrupted. Note that a significant portion of the model lies within the pair-instability regime marked by the thick red curve.

For all of the models considered here the final, pre-SN mass of the CO core remnants $\left(M_{\mathrm{CO}}\right)$ was in the range of $\sim 60-112 M_{\odot}$ and the final radius, $R_{\mathrm{f}}$, in the range $4 \times 10^{10}-4 \times 10^{13} \mathrm{~cm}$. The rotating models consistently made more compact PISN progenitors due to the effects rotationally enhanced mass-loss. Due to angular momentum transport during the main evolution, the final, pre-SN rotation rates of the $\mathrm{CO}$ cores were somewhat modest $\left(\Omega / \Omega_{\mathrm{c}} \simeq 0.02-0.06\right)$ with the exception of the $140 M_{\odot}$ no-ST model. As such, most of these pre-SN models have somewhat different structural properties than other PISN models considered so far in the literature for radiative transfer studies (Gal-Yam et al. 2009; Kasen et al. 2011; Dessart et al. 2013). 


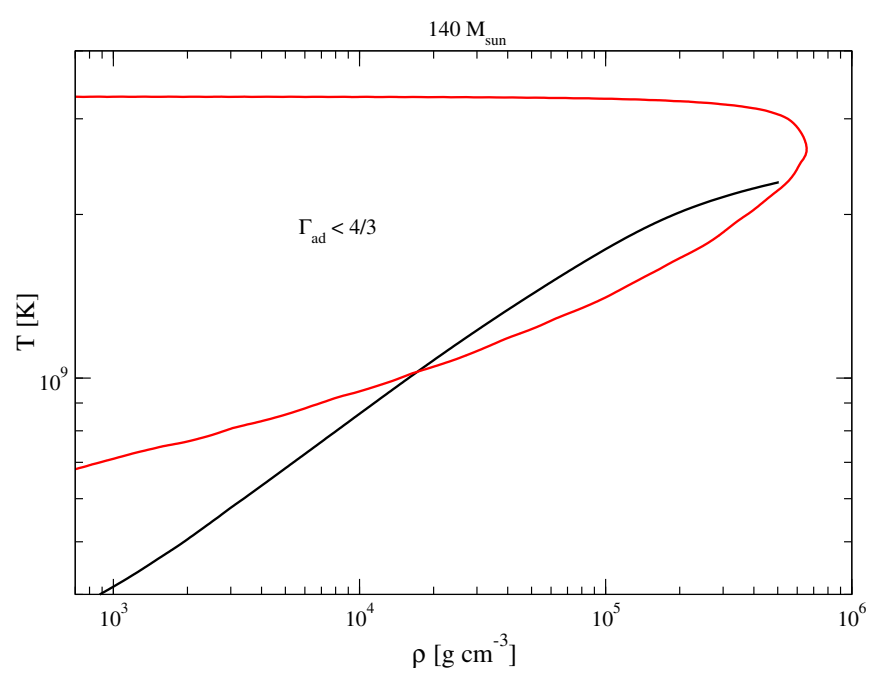

Figure 1. Black curve shows the initial MESA progenitor $\rho-T$ profile for the $140 M_{\odot}$ model prior to mapping to the grid of FLASH. The thick red curve denotes the region where $\Gamma_{\mathrm{ad}}<4 / 3$ due to the effects of $\mathrm{e}^{-}-\mathrm{e}^{+}$pair production.

They are compact, well chemically mixed slowly rotating CO stars with the $\mathrm{H}$ envelope removed in all cases and with a low, even zero in a few cases, He envelope mass.

\subsection{ID FLASH Hydrodynamics}

All the pre-SN 1D profiles from MESA were mapped to the 1D AMR Eulerian grid of FLASH 4.0 (Fryxell et al. 2000; Dubey et al. 2009). The same basic physics units (equation of state, nuclear reaction network) are used in FLASH as in the MESA stellar evolution calculations with the exception that we did not map the pre-SN rotational profile for the hydrodynamics simulation. As Chatzopoulos et al. (2013a) have shown, when the magnetic viscosity and angular momentum transport due to the ST dynamo action are included in the stellar evolution calculation, the pre-SN rotation is slow and does not affect the final hydrodynamics. It is the pre-SN mixing changing the structure of the progenitor star and the composition gradient effects that primarily determine the final dynamics and nucleosynthetic properties of the explosion. Also, the oblateness of the star due to rotation will be negligible for the moderate pre-SN rotational velocities of our suite of models.

We follow the collapse, explosion, and nucleosynthesis in FLASH up until prior to SN SBO, which we take to be the phase when the $\mathrm{SN}$ shock is within a hundred to a few thousand optical depths from the surface of the star, still in the optically thick regime. We recall that $\tau_{\mathrm{SBO}}=c / v_{\mathrm{sh}}$ where $\tau_{\mathrm{SBO}}$ is the $\mathrm{SBO}$ optical depth, $v_{\mathrm{sh}}$ the velocity of the $\mathrm{SN}$ shock (typically of the order of a few $10^{9} \mathrm{~cm} \mathrm{~s}^{-1}$ ), and $c$ the speed of light. Figure 2 shows the composition (upper panel), velocity (middle panel), and density (lower panel) for the pre-SBO stage when we halt the FLASH simulation in the case of the $140 M_{\odot}$ model. As illustrated in Figure 2, the basic structure of this model consists of ${ }^{56} \mathrm{Ni}$ located in the base of H-poor SN ejecta. Table 1 details the basic characteristics of all PISN explosion models. Our set of models produced final ${ }^{56} \mathrm{Ni}$ masses in the range $0.1-22 M_{\odot}$ embedded in SN ejecta of $60-112 M_{\odot}$. The $M_{\mathrm{Ni}} / M_{\mathrm{f}}$ ratios for our rotating 90-140 $M_{\odot}$ and non-rotating $200 M_{\odot}$ and $260 M_{\odot}$ PISN models are consistent with those of Dessart et al. (2013) meaning that, modulo differences in SN ejecta opacity, our models are expected to manifest as dimmer events (in terms

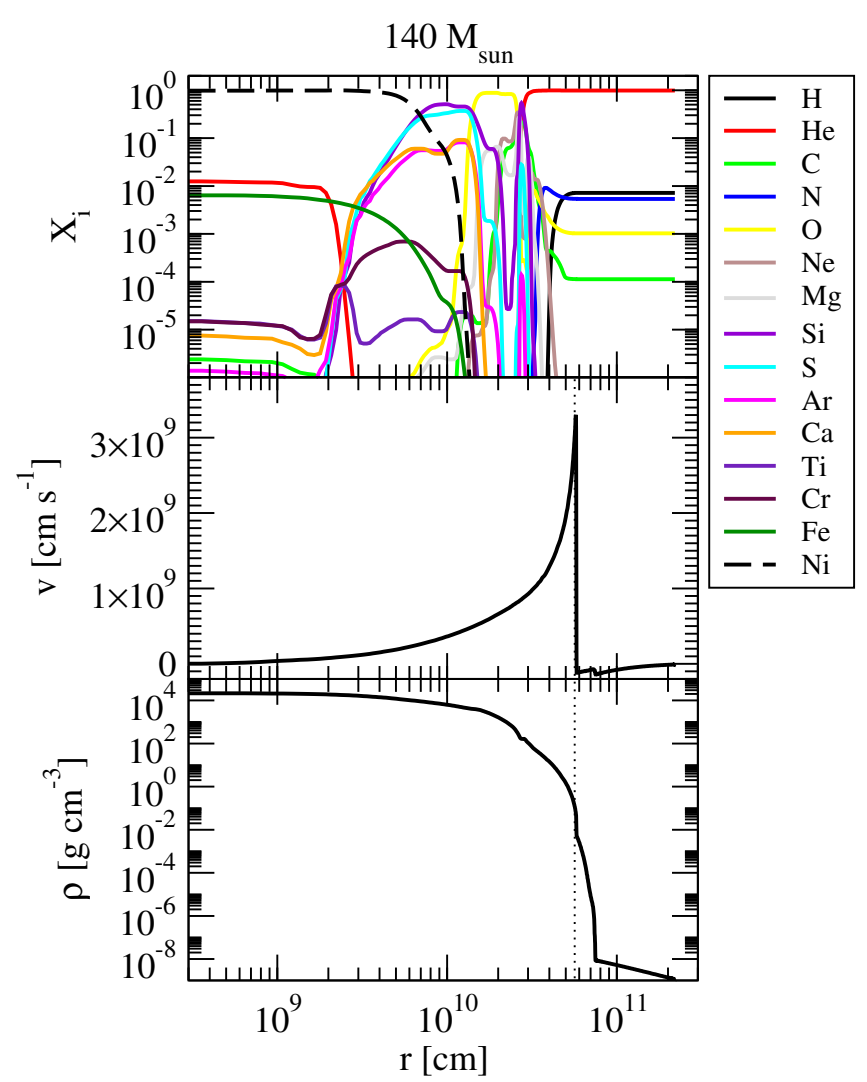

Figure 2. 1D FLASH radial profiles of composition (upper panel), velocity (middle panel), and density (lower panel) for the $140 M_{\odot}$ model prior to mapping to the grid of $R A G E$ just before SN shock breakout. The dotted vertical lines correspond to the location of the SN shock.

of bolometric LC) that have rather red colors. We test this prediction in Section 4.

\section{ROTATING PISN SHOCK BREAKOUT EMISSION}

As an intermediate step, the 10 final rotating zero-metallicity PISN FLASH profiles were mapped into the 1D AMR Eulerian grid of the radiation hydrodynamics code $R A G E$ (Gittings et al. 2008; Frey et al. 2013) where the emission during the SBO phase is modeled. We did not map the other models into $R A G E$ because higher metallicity and non-rotating PISN SBO properties have already been well studied in the literature (Whalen et al. 2013a, 2013b). The SBO LCs are calculated by post-processing the $R A G E$ snapshots with the LANL code SPECTRUM (Frey et al. 2013). For details on the physics, resolution and other parameters used in RAGE and SPECTRUM please see Whalen et al. (2013b).

The RAGE and SPECTRUM results on the ${ }^{56} \mathrm{Ni}$ radioactive decay powered re-brightening seem to conflict with earlier results and a code comparison effort is underway to determine the source of this discrepancy (Whalen et al. 2013a). Possible sources include differences in opacities, resolution, and the issue of what fraction of the radioactive decay energy goes into increasing the kinetic energy of the inner SN ejecta versus pure radiative losses. Homologous LTE and non-LTE codes are engineered to predict behavior closer to the latter while $R A G E$ radiation hydrodynamics favors the former case. In this paper, we are only using $R A G E$ to model the SBO emission during the first few days of radiation hydrodynamical evolution while for 

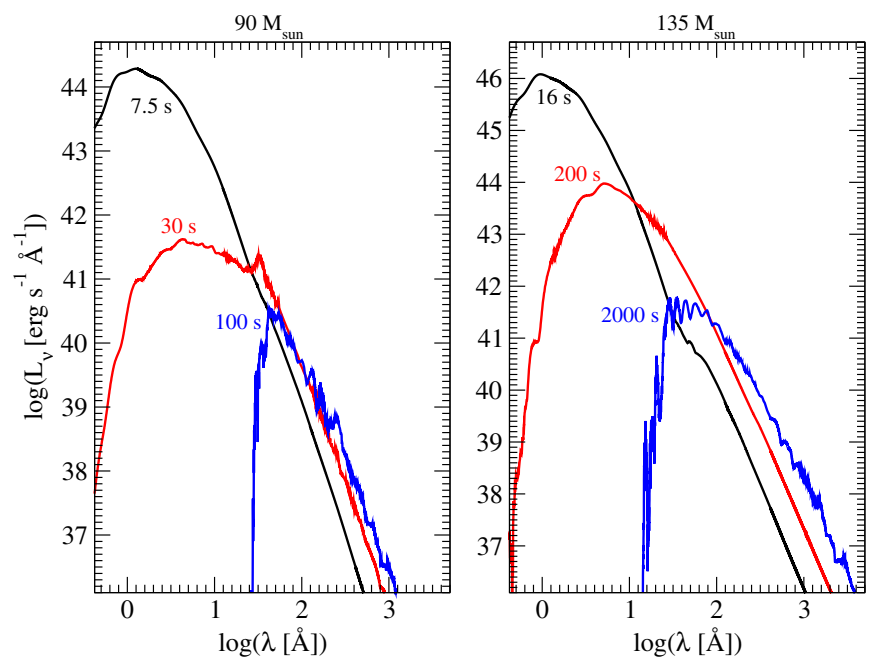

Figure 3. Post-SBO SED evolution for the $90 M_{\odot}$ (left panel) and the $135 M_{\odot}$ (right panel) model as calculated by SPECTRUM. The SED phases are shown on top of the corresponding curves. The SBO emission for a bare $\mathrm{CO}$ rotating PISNe peaks in the hard X-rays (0.1-10 ̊̊ or $\sim 1-100 \mathrm{keV})$.

the later evolution (homologous expansion phase) we use the LTE treatment of the PHOENIX code (Section 4).

The SBO LCs have some dependence on the properties of the CSM that is superimposed on the SN blast profiles in the $R A G E$ grid. For the purposes of this study, the abrupt density gradient between the edge of the star and the wind is bridged by an $r^{-20}$ profile to minimize effects of numerical instabilities during the SBO phase. Then the wind profile follows an inverse square power-law given explicitly by $\rho_{w}=\dot{M} /\left(4 \pi v_{w} r^{2}\right)$ where $\rho_{w}$ is the wind density, $\dot{M}$ the wind mass-loss rate, and $v_{w}$ the wind velocity taken to be $1000 \mathrm{~km} \mathrm{~s}^{-1}$. For all models we fix the density at the bottom of the wind profile to be $\sim 2 \times 10^{-18} \mathrm{~g} \mathrm{~cm}^{-3}$ so that the CSM is optically thin.

Using SPECTRUM, the spectral energy distributions (SEDs) during the first few days after SBO are calculated and then integrated yielding the final bolometric LCs. In Figure 3 the first few minutes of post-SBO SED evolution for the $90 M_{\odot}$ and the $135 M_{\odot}$ models are shown. The SBO spectra for all of our models exhibit a characteristic "expanding fireball" behavior that is well described by a cooling blackbody continuum with some spectral lines superimposed, especially during the later phases. The peak of the emission during SBO is in the hard X-ray part of the spectrum $(0.1-10 \AA$ or $\sim 1-100 \mathrm{keV})$ at significantly shorter wavelengths than SBO emission from red supergiant (RSG) and blue supergiant (BSG) PISN progenitors (Kasen et al. 2011).

Figure 4 shows the resulting SBO bolometric LCs (solid curves) for all of the models discussed in this paper. The late-time PHOENIX LCs are also shown (dashed curves) for completeness but discussed in detail in Section 4. The peak SBO luminosities for all models are in the range $10^{45}-10^{46} \mathrm{erg} \mathrm{s}^{-1}$ and are consistent with the Kasen et al. (2011) results despite the different progenitor properties. This is mainly because although our post-SBO temperatures and velocities are higher and the emission peaks at lower wavelengths, the radii of our progenitor models are smaller than both the RSG and BSG series presented in Kasen et al. (2011). The rotating PISN SBO bursts are also found to be close in terms of duration with those for BSG progenitors in Kasen et al. (2011). After the end of the SBO bursts (0.01-0.1 days), the luminosities are mainly driven by hydrodynamical effects (reverse and forward shock
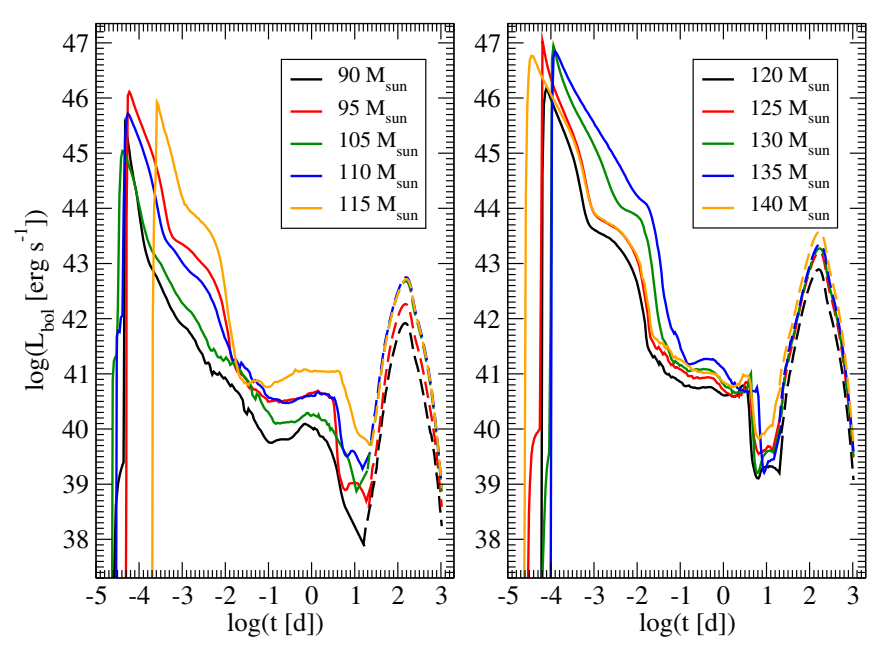

Figure 4. RAGE/SPECTRUM SBO (solid curves) and PHOENIX late-time (dashed curves) LCs for the grid of rotating PISNe models studied here. Left Panel: $90-115 M_{\odot}$. Right Panel: $120-140 M_{\odot}$.

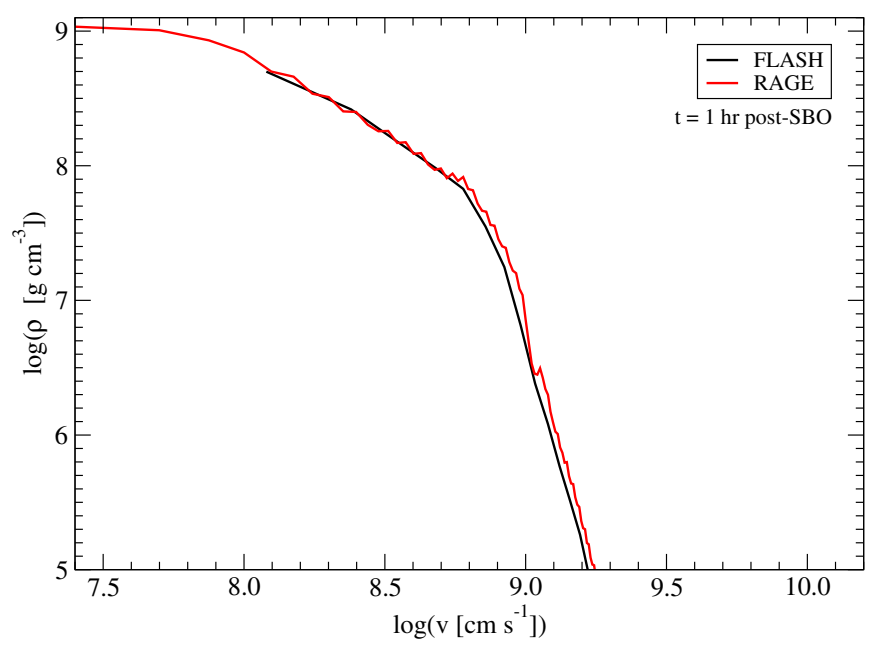

Figure 5. Scaled density profile in Lagrangian coordinates at $1 \mathrm{hr}$ after SBO in FLASH (black curve) and RAGE (red curve). It can be seen that the pure hydrodynamics and radiation hydrodynamics profiles are in good agreement shortly after SBO as discussed in Section 4.

heating) before the much longer re-brightening phase due to the radioactive decays of ${ }^{56} \mathrm{Ni}$ and ${ }^{56} \mathrm{Co}$ starts.

\section{TIME-DEPENDENT RADIATIVE TRANSFER}

In order to calculate time-dependent model spectra for our rotating PISN models we first converted the blast profiles from FLASH at $1 \mathrm{hr}$ after SBO into the Langragian grid using velocity as the independent variable. These profiles are then input to the radiative transfer code $P H O E N I X$ (Hauschildt 1992; Hauschildt \& Baron 1999, 2004; van Rossum 2012a), allowing us to retrieve model light curves, color, and spectral evolution.

At first, it might seem questionable that we elected to use the FLASH post-SBO profiles instead of the RAGE ones that properly model the radiation hydrodynamic effects during that early phase; however, once the SBO effects have ceased we find that the density and mass fraction profiles calculated by the two codes are almost identical up to $\sim 1-4$ days, depending on the model. To illustrate this we present the Lagrangian density profiles for the $140 M_{\odot}$ model at $1 \mathrm{hr}$ in Figure 5 for both $F L A S H$ (black curve) and RAGE (red curve). We have verified that the resulting final model LCs and spectra are virtually invariable 

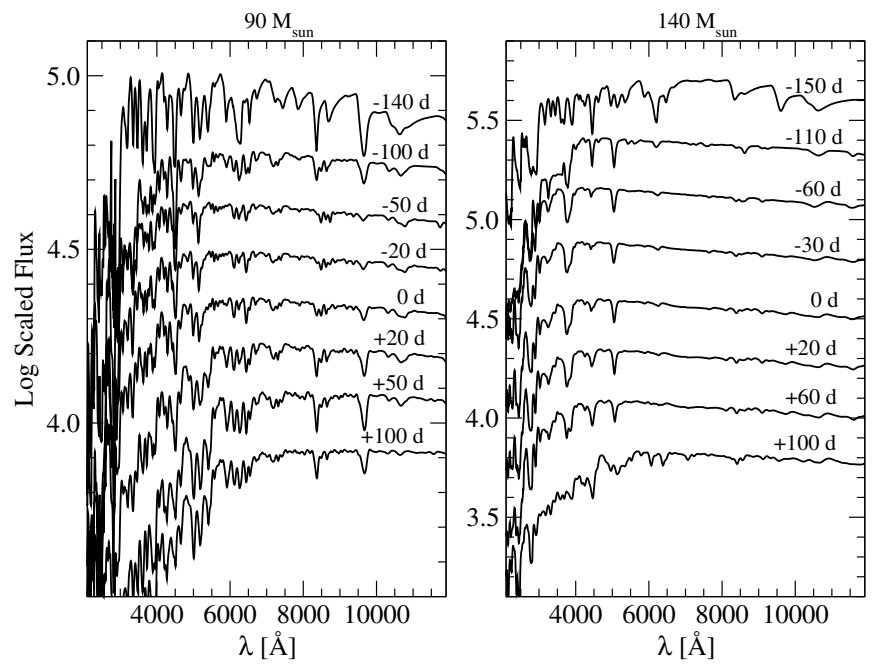

Figure 6. $P H O E N I X$ spectral evolution for the $90 M_{\odot}$ (left panel) and the $140 M_{\odot}$ (right panel) models. The phases are shown with respect to the time of ${ }^{56} \mathrm{Ni}$ decay peak luminosity.

with respect to what input profile is used at $1 \mathrm{hr}$ after SBO (FLASH or RAGE).

PHOENIX solves the special relativistic radiative transfer problem in spherical symmetry using the highly accurate short characteristics and operator splitting methods (Olson \& Kunasz 1987). The time-evolution of the post-hydrodynamic, radiationdominated phase is followed using the Radiation Energy Balance method (van Rossum 2012a). PHOENIX does not use the Sobolev approximation, diffusion approximations, or opacity binning. The deterministic nature of the radiative transfer method enables calculation of noise-free spectra, a feature which enables us to show detailed line identifications for the presented model spectra (see next paragraph).

\subsection{Zero-metallicity $90-140 M_{\odot}$ PISNe}

The spectral evolution of the lower mass $\left(90 M_{\odot}\right)$ and the higher mass $\left(140 M_{\odot}\right)$ rotating PISN is shown in Figure 6, where the phases cited are with respect to the time of peak luminosity during the later phase of ${ }^{56} \mathrm{Ni}$ heating of the ejecta. The hotter, more massive models are less line-dominated than the less massive ones and exhibit a stronger continuum due to their higher temperatures. To better illustrate the spectroscopic characteristics of rotating PISNe we also show only the peak luminosity spectra for all models in Figure 7 as well as detailed spectral line identifications in Figure 8 for the 90 and $140 M_{\odot}$ models.

The spectral line identification shown in Figure 8 was performed by removing the line-opacity from individual ions, rerunning the PHOENIX models and comparing with the original spectrum. The difference between each of the "knock-out" spectra and the original is attributed to the lines of the respective ion (van Rossum 2012b). In Figure 8 we stack the differences in flux level from different runs on top of each other, using distinct colors for different chemical elements. Colored patches on top of the original flux level are caused by (the absence of) absorption, and patches below the original flux level by (the absence of) emission. The plots show that some of the lines that appear in the spectra cannot be fully attributed to a single chemical element but rather to a combined effect of multiple species.

Figures 6 through 8 reveal that the optical spectra of all rotating PISN spectra are dominated by intermediate mass elements (IMEs). More specifically, the early to peak luminosity
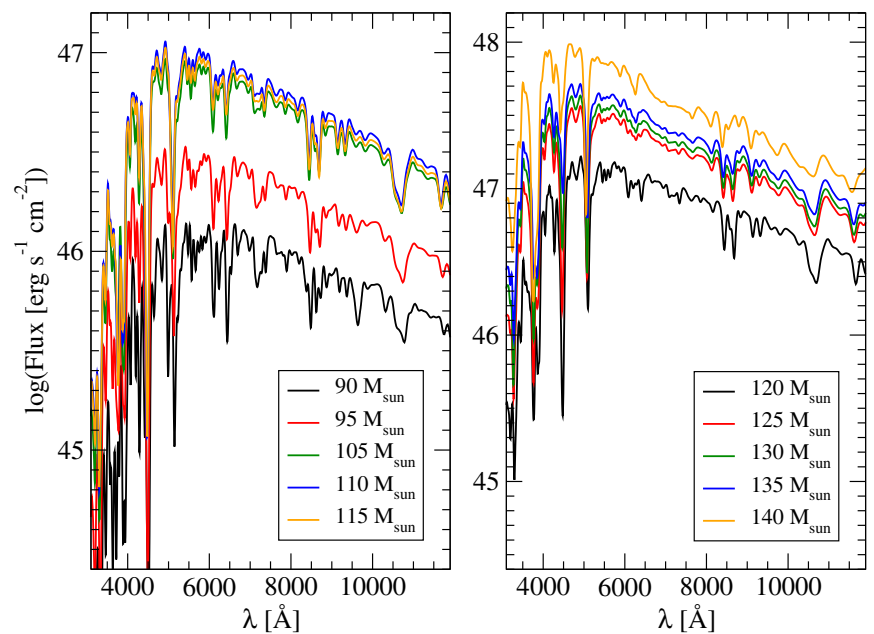

Figure 7. $P H O E N I X$ spectra at peak luminosity for the zero-metallicity rotating 90-140 $M_{\odot}$ PISNe. Left panel: $90-115 M_{\odot}$. Right panel: 120-140 $M_{\odot}$.

spectra are dominated by strong $\mathrm{Mg}, \mathrm{Si}$, and $\mathrm{Ca}$ lines with some iron-group elements present in the near-UV (mostly $\mathrm{Cr}$ and $\mathrm{Ti}$ ). The strong $\mathrm{Mg}$ absorption is due to the high abundance of $\mathrm{Mg}$ in the middle layer of the PISN ejecta (for example see the upper panel of Figure 2). Enhanced Mg in PISN ejecta is also seen by other studies (Heger \& Woosley 2002; Woosley et al. 2007) and it is the result of the passage of the burning flame from this region before it shuts off prior to reaching the outer ejecta. The $6000 \AA<\lambda<8000 \AA$ domain is virtually devoid of prominent line features in the more massive model (with the exception of the Si $\lambda 6150 \AA)$. In addition, all models show prominent Si absorption features in the near-IR $(\lambda>10500 \AA)$.

Although the SN ejecta had considerable amounts of $\mathrm{He}$, no significant $\mathrm{He}$ features are seen in any of the spectra due to the fact that the photosphere recedes quickly through the $\mathrm{He}$ envelope but also due to the low envelope temperatures that are insufficient for low atomic level excitation and generation of optical lines. We caution, however, that non-LTE treatment will likely result in the presence of some He features due to excitation by secondary electrons produced by Compton scattering of the gamma rays produced by the radioactive decays of ${ }^{56} \mathrm{Ni}$ and ${ }^{56}$ Co (Lucy 1991; Hachinger et al. 2013). Over time as the expanding SN ejecta cool and the photosphere recedes into the inner regions additional lines appear characteristic of the inner ejecta ( $\mathrm{Ti}, \mathrm{Cr}, \mathrm{Fe}, \mathrm{Co}$; bottom panels of Figure 8). The basic spectral characteristics are very similar to those observed in the He100 models of Kasen et al. (2011) and Dessart et al. (2013).

Convolution of the spectra with the standard UBVRIJHK broadband filters allows for the calculation of photometric LCs that are shown in Figure 9 for the $90 M_{\odot}$ and the $140 M_{\odot}$ models. In a manner characteristic of regular SN events, the redder LCs are brighter and with longer diffusion timescales. Integration of the spectra yields the final bolometric LCs for all of the rotating PISN models studied here, shown in Figure 10. The peak bolometric luminosities are in the range $10^{43}-5 \times 10^{44}$ (corresponding to bolometric magnitudes in the range $-16 \lesssim$ $M_{\text {bol }} \lesssim-20$ ) while the rise time to peak luminosity is in the range 140-170 days in all cases. As such, none of these models are as bright as typical SLSN events $\left(M_{\mathrm{bol}}>-21 \mathrm{mag}\right.$; Gal-Yam 2012). Rotating PISNe luminosities span the whole range that is characteristic of other, regular luminosity events such as Type IIP, Type IIb/c, and regular Type IIn events and 

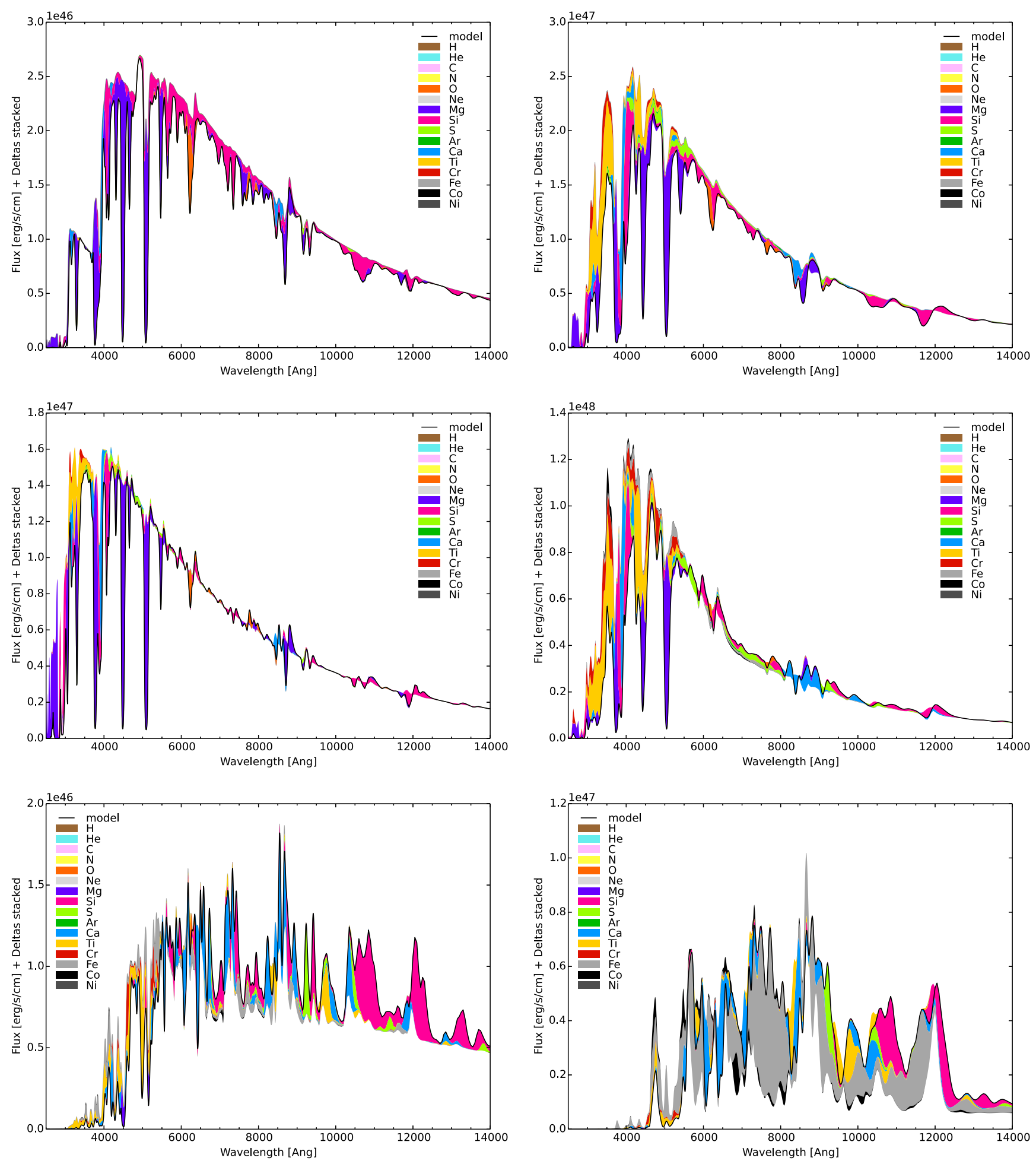

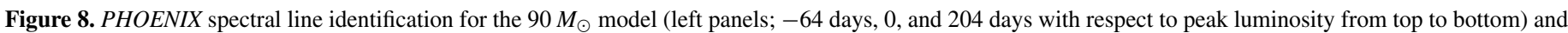

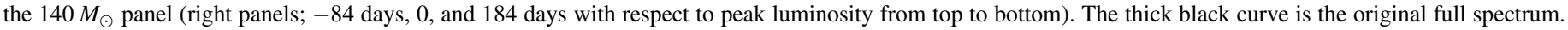
For a more detailed interpretation of this plot refer to the discussion in Section 4.1.

can also bridge the gap between regular CCSNe and SLSNe. Rotating PISNe of much larger mass, however, can possess peak luminosities characteristic of SLSNe (Section 4.2). A distinguishing characteristic of PISN LCs as compared to LCs of other SN events is obviously the much slower timescales over which they evolve.
Another characteristic of PISN based upon our analysis is their intrinsically red color regardless of rotation. Figure 11 shows the $B-V, B-R, V-I$, and $V-K$ color evolution for the $90 M_{\odot}$ and the $140 M_{\odot}$ models. All of our models remain red in color, more so during their post-peak luminosity evolution. As expected, lower mass PISNe are redder than higher mass 

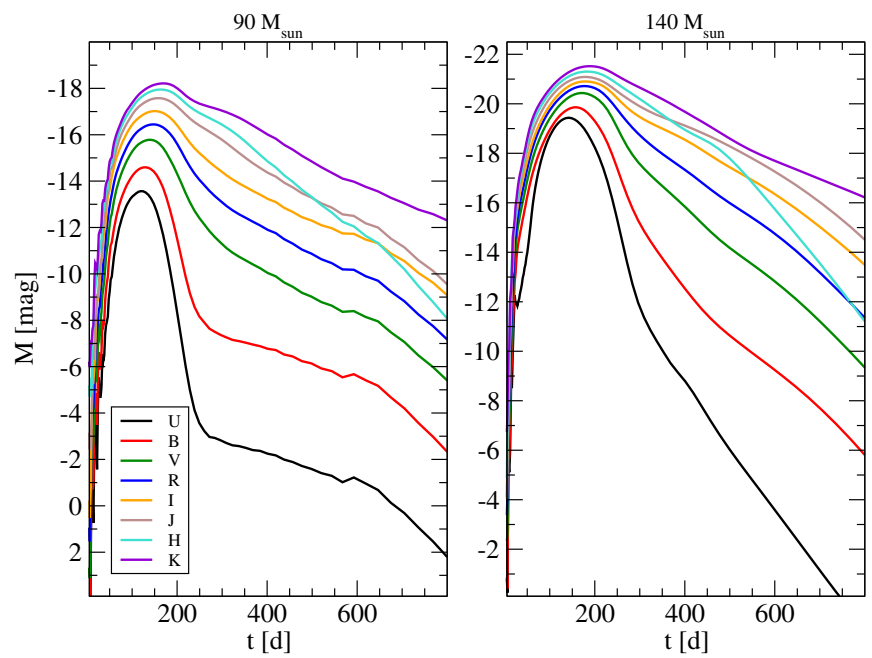

Figure 9. Broadband UBVRIJHK PHOENIX LCs for the $90 M_{\odot}$ (left panel) and the $140 M_{\odot}$ (right panel) models. Note how the SN LC is dimmer and has a steeper post-maximum decline in shorter wavelengths.
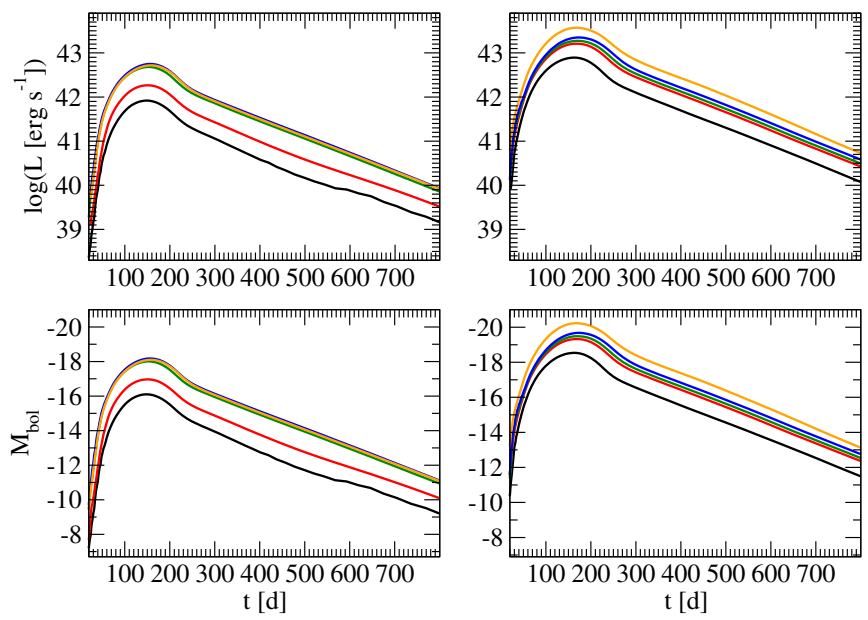

Figure 10. PHOENIX LCs in terms of luminosity (upper panels) and absolute bolometric magnitude, $M_{\text {bol }}$ (lower panels) for the zero-metallicity 90-140 $M_{\odot}$ PISNe. None of these events is superluminous and all peak luminosities are within the range of those of regular core-collapse SNe. The colors of the curves correspond to the same models as in Figure 4.
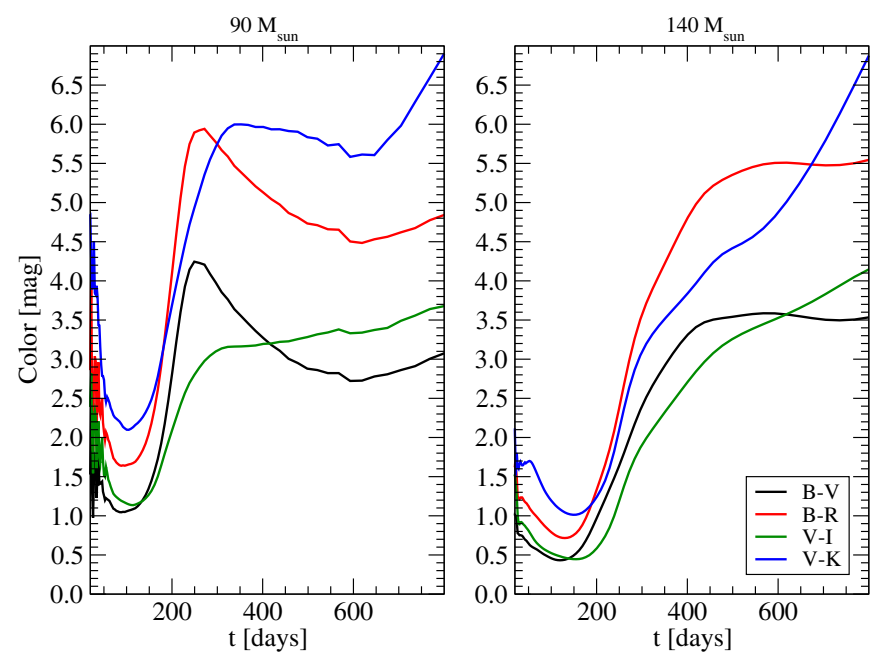

Figure 11. Evolution of the $B-V, B-R, V-I$, and $V-K$ colors for the $90 M_{\odot}$ (left panel) and the $140 M_{\odot}$ (right panel) model. The color evolution of PISNe is redder than that of other types of $\mathrm{SNe}$.
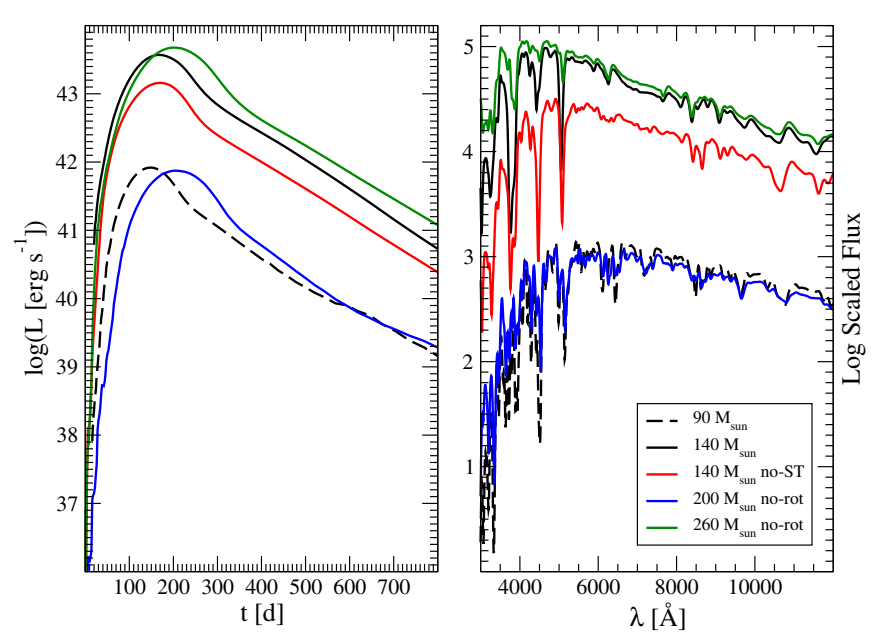

Figure 12. Bolometric LCs (left panel) and spectra (right panel) of the rapidly rotating $140 M_{\odot}$ non-magnetic (no-ST) model (red curve) and the non-rotating 200 and $260 M_{\odot}$ models (blue and green curves, respectively; Section 4.2) compared to the original $90 M_{\odot}$ and $140 M_{\odot}$ models (solid and dashed black curves, respectively).

events. This is also in agreement with the results of Dessart et al. (2013).

\subsection{Effects of Rotation and Metallicity}

As discussed in Section 2.1, in order to assess the effects of rotation to the radiative properties of PISN, we have run a rapidly rotating model without the effects of the ST dynamo $\left(140 M_{\odot}\right.$, no-ST $)$ and two non-rotating models $\left(200 M_{\odot}\right.$ and $\left.260 M_{\odot}\right)$ all at zero-metallicity. The main properties of these models are presented in Table 1.

Prior to examining how LCs and spectra compare between progenitors with different ZAMS rotation rates, we summarize the main effects of rotation on the structure of PISN progenitors discussed by Chatzopoulos \& Wheeler (2012a). Rotationally induced mixing (mainly due to meridional circulation and the ST mechanism) chemically homogenizes the star and enhances production of $\mathrm{C}, \mathrm{N}$, and $\mathrm{O}$. In addition, rotationally induced mass-loss and enhanced radiatively driven mass-loss due to the increased presence of metals in the outer layers leads to the loss of all of the $\mathrm{H}$ and most (or in some cases all) of the He envelope. In the case of zero rotation, we find that PISN progenitors retain both $\mathrm{H}$ and $\mathrm{He}$ and have RSG-type large radius envelopes. The CO core structures of the stars are, however, very similar regardless of the initial rotation. As a result, once the PISN sets in the nucleosynthetic products of the explosion are similar in all progenitors and the structures imported in PHOENIX quite similar, regardless of the initial rotation rate. The only notable differences are the increased surface abundances of $\mathrm{C}, \mathrm{N}$, and $\mathrm{O}$ for the rotating models.

For the compact rotating PISN, the photospheric temperatures remain very high over timescales past peak luminosity ( $>10,000 \mathrm{~K}$ ) keeping $\mathrm{H}, \mathrm{He}, \mathrm{C}, \mathrm{N}$, and $\mathrm{O}$ ionized. This fact, together with the intrinsically weak nature of the $\mathrm{C}$ and $\mathrm{N}$ lines, hints that we should not expect strong lines indicative of the increased $\mathrm{C}, \mathrm{N}$, and $\mathrm{O}$ surface abundances in the model spectra of the rotating models. Figure 12 showing bolometric LCs (left panel) and peak luminosity spectra (right panel) for models of different ZAMS rotation rates confirms this assertion.

We observe that the bolometric LCs of the $90 M_{\odot}$ and the $200 M_{\odot}$ models and those of the $140 M_{\odot}$ and the $260 M_{\odot}$ models have similar peak luminosities (albeit different diffusion 

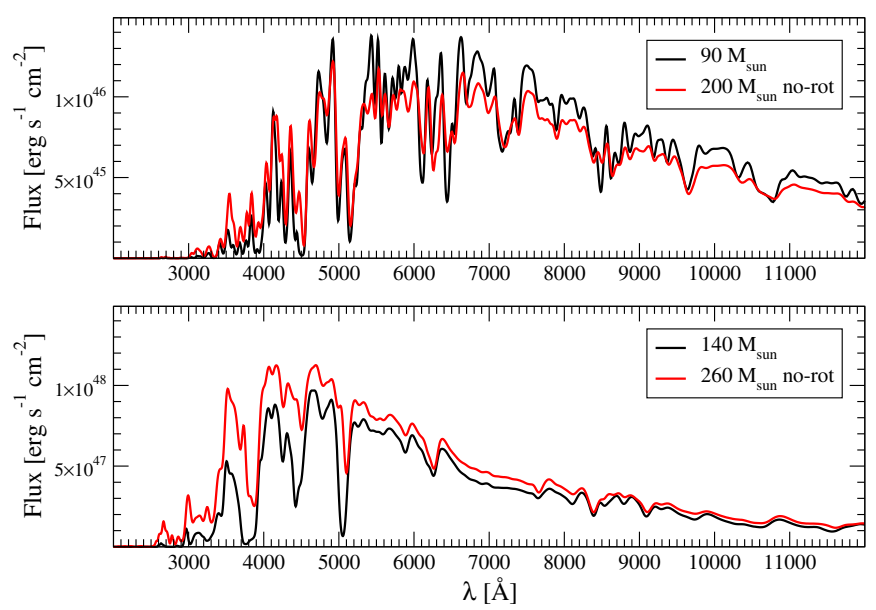

Figure 13. Peak spectrum comparisons for the $90 M_{\odot}$ and the $200 M_{\odot}$ no-rot (upper panel) and the $140 M_{\odot}$ and the $260 M_{\odot}$ (lower panel) models. The effects of pre-SN rotation do not have observable effects on the resulting spectra of PISN with similar $M_{\mathrm{CO}}$ and bolometric LC properties. The enhanced mass-loss suffered by the $90 M_{\odot}$ model accounts for the more pronounced differences (mainly the O I $\lambda 6454 \AA$ and $\lambda 7777 \AA$ absorption features).

timescales) and we will therefore focus our spectroscopic comparison efforts to these two groups of models. A more clear comparison of the peak spectra for these two sets of models is shown in the lower panel of Figure 13. The main spectral features of the $140 M_{\odot}$ and $260 M_{\odot}$ models are very similar considering the different peak bolometric luminosities. Strong $\mathrm{Si}, \mathrm{Mg}$, and $\mathrm{Ca}$ features are seen in the optical with $\mathrm{Mg}$ and irongroup line blends strongly present in the near-UV. Regardless of the intrinsic differences in the temperature and the velocity of the expanding SN photospheres (leading to differences in the line fluxes in the 3000-5000 $\AA$ region), there are no clear indicators of pre-SN rotation present in these spectra. The spectra at earlier phases also appear to be very similar between the two models.

In the $90 M_{\odot}$ versus $200 M_{\odot}$ case there are a few notable differences attributable to the pure $\mathrm{CO}$ core structure of the $90 \mathrm{M}_{\odot}$ PISN. mass-loss led to the total loss of the $\mathrm{H}$ and He envelope for this model leaving an oxygen-rich progenitor star prior to explosion. In contrast, the outer layers of the $200 M_{\odot}$ model were still $\mathrm{H}$ and $\mathrm{He}$ rich. As a result the peak luminosity spectrum of the $90 M_{\odot}$ model shows stronger O I absorption features at $\lambda 6454 \AA$ and $\lambda 7777 \AA$. These subtle differences can be more clearly distinguished in the upper panel of Figure 13. For better intuition we show the $\mathrm{SN}$ ejecta compositions of the rotating and non-rotating models compared in Figure 14. In can be seen that the more massive models (right panels; $140 M_{\odot}$ and $260 M_{\odot}$ ) have similar SN ejecta composition profiles regardless of rotation while the lower mass models have differences in the outer ejecta with the $90 M_{\odot}$ model having an O-rich outer envelope.

The color evolution for the models of different ZAMS rotation rates discussed here is shown in Figure 15. A comparison of this figure with Figure 11 indicates that, based on the color properties alone, one cannot see a clear distinction between otherwise similar rotating and non-rotating PISN.

To infer the effects of rotation on the radiative properties of PISN, and more specifically on spectra, one has to be cautious as to not over-interpret the observations. The features in the optical part of the spectrum are more robust than the ones in the near-UV where $\mathrm{Mg}$ but also a variety of iron-group elements ( $\mathrm{Ti}, \mathrm{Cr}, \mathrm{Fe}$ ) contribute to several line-blends. Even in the case of the O I features present in the model spectrum of the $90 M_{\odot}$ PISN, it would be unfounded to attribute them solely to the effects of rotation since mass-loss was the primary reason the progenitor star was mostly composed of pure oxygen. Although
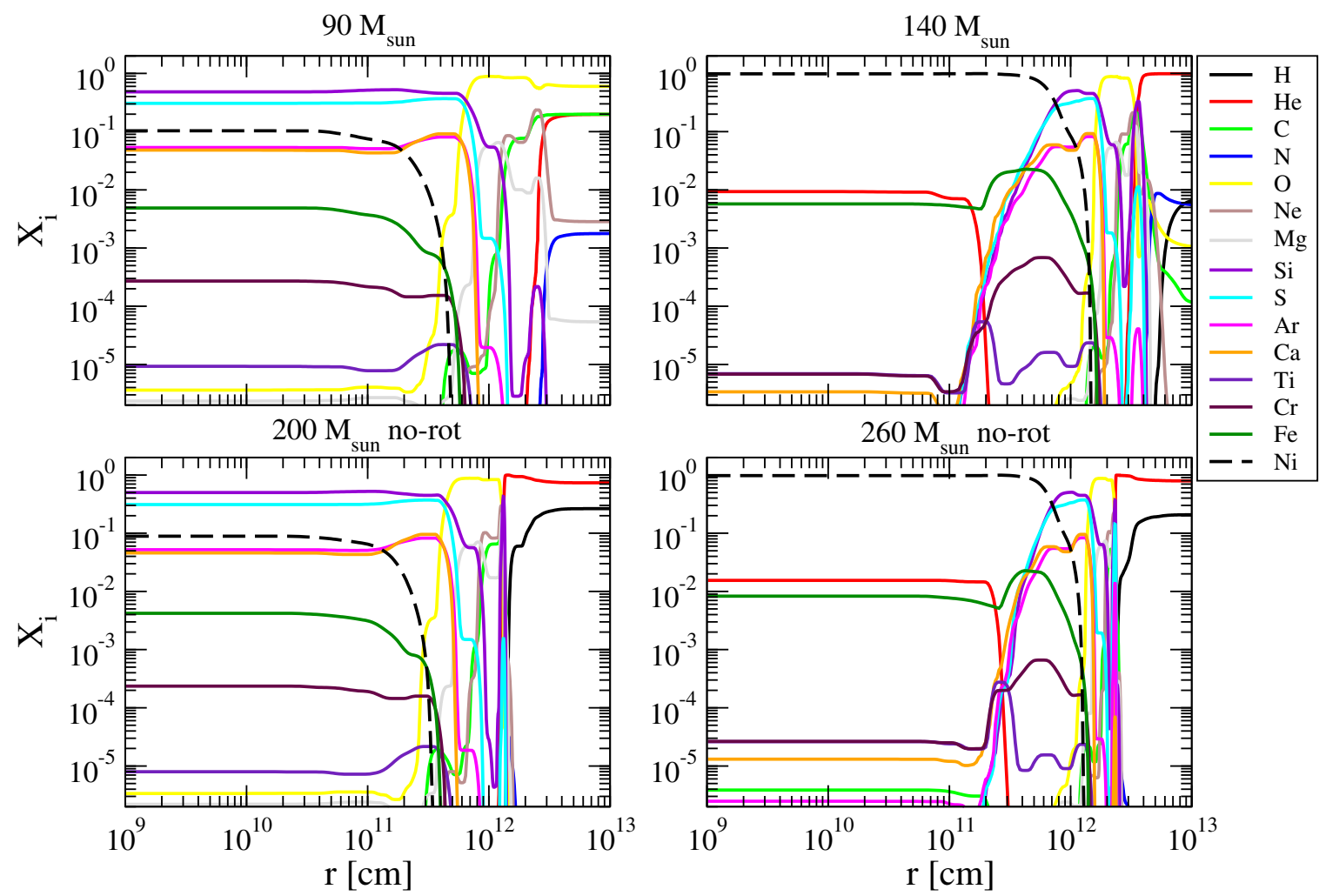

Figure 14. Comparison of the pre-SN compositions of the rotating $90 M_{\odot}$ and $140 M_{\odot}$ models (upper panels) against the non-rotating $200 M_{\odot}$ and $260 M_{\odot}$ models (lower panels) that produce similar bolometric LCs (Figure 12). 

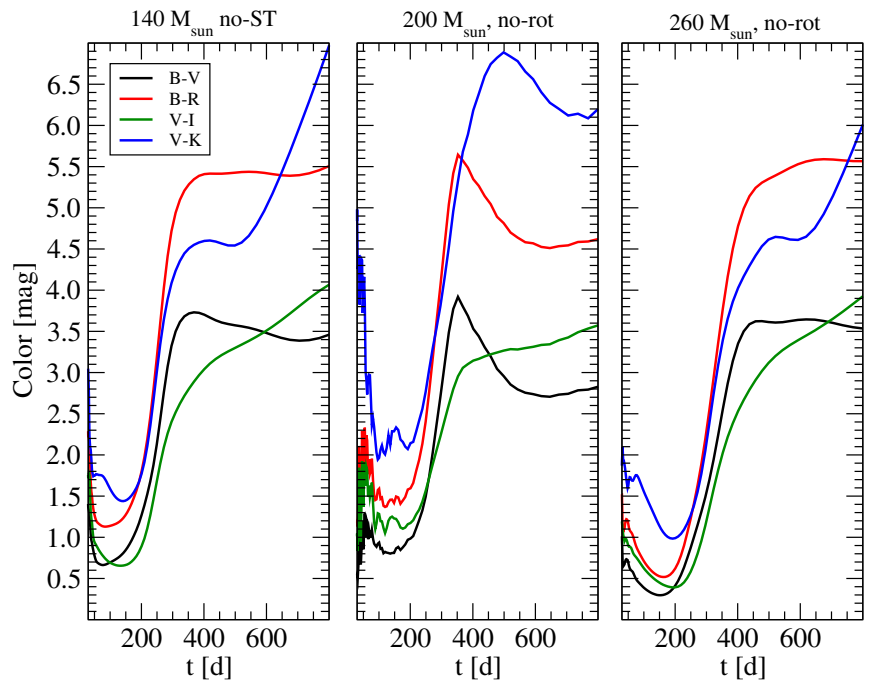

Figure 15. Evolution of the $B-V, B-R, V-I$, and $V-K$ colors for the $140 M_{\odot}$ non-magnetic (no-ST; left panel), the $200 M_{\odot}$ no-rot (middle panel), and the $260 M_{\odot}$ no-rot (right panel) model (Section 4.2).
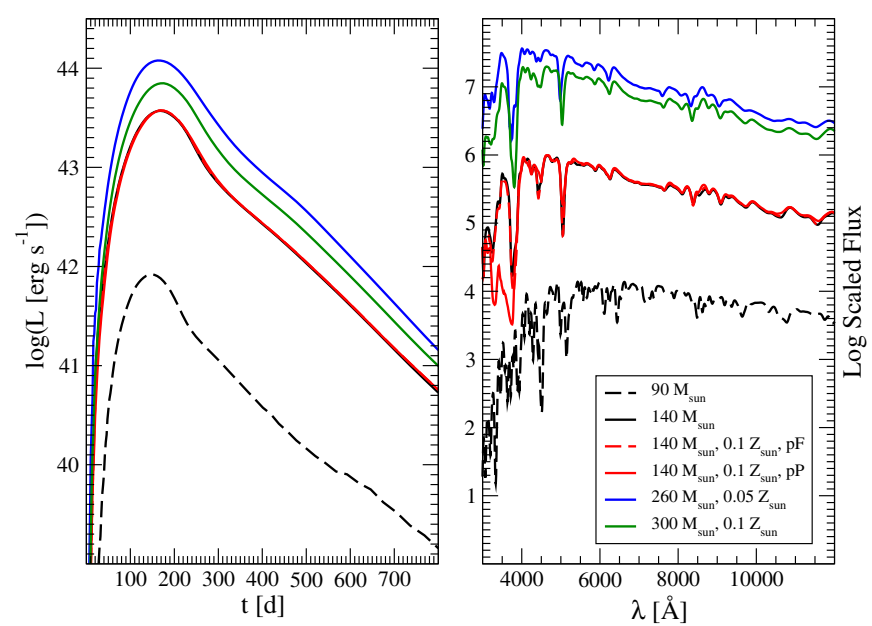

Figure 16. Bolometric LCs (left panel) and spectra (right panel) of the $0.1 Z_{\odot}$ $140 M_{\odot}$ model prior to import to FLASH ("pF"; dashed red curve) and PHOENIX ("pP"; solid red curve), and the $0.05 Z_{\odot} 260 M_{\odot}$ and the $0.1 Z_{\odot}$ $300 M_{\odot}$ models (blue and green curves, respectively) compared to the original $90 M_{\odot}$ and $140 M_{\odot}$ models (solid and dashed black curves, respectively). Evolution with different initial metallicity does not change the resulting PISN spectra significantly. Artificial addition of metal content prior to the radiative transfer calculation leads to the suppression of UV flux (model "pP").

rotation is one avenue of mass-loss enhancement there are alternative possibilities (binary interaction, episodic/luminous blue variable (LBV-type) mass-loss). Also, differences in the SN blast profiles (in terms of temperature and velocity) further increase the degeneracy between non-rotating and rotating PISN spectra. Perhaps the only qualitative difference between rotating and non-rotating models is the apparent suppression of the nearUV flux $(\lambda<3500 \AA)$ attributable to the increased presence of metals in the rotating case due to the effects of pre-SN mixing. Conversely, this effect could also be due to intrinsically higher metallicity, regardless of rotation (Kasen et al. 2011).

To study the effects of metallicity on the radiative properties of PISNe we have run the models $0.05 Z_{\odot} 260 M_{\odot}, 0.1$ $Z_{\odot} 300 M_{\odot}$, and $0.1 Z_{\odot} 140 M_{\odot}$ as described in Section 2.1 and Table 1. Figure 16 shows the resulting PHOENIX bolometric LCs (left panel) and spectra (right panel) as compared to our base zero-metallicity $90 M_{\odot}$ and $140 M_{\odot}$ models. Fig-
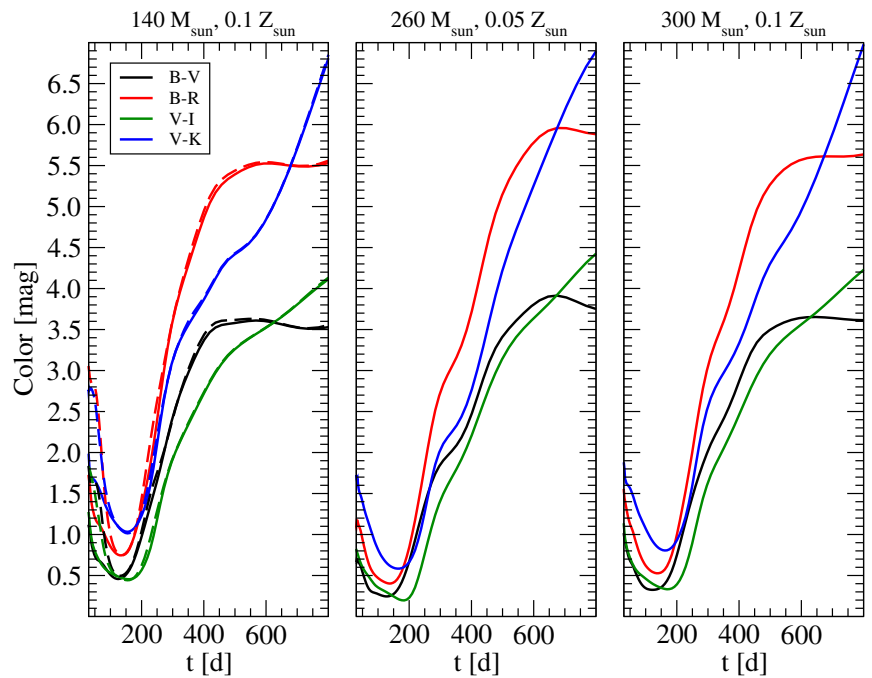

Figure 17. Evolution of the $B-V, B-R, V-I$, and $V-K$ colors for the $0.1 Z_{\odot}$ $140 M_{\odot}$ (left panel), the $0.05 Z_{\odot} 200 M_{\odot}$ (middle panel) and the $0.1 Z_{\odot}$ $300 M_{\odot}$ (right panel) model (see Section 4.2). The dashed curves in the left panel correspond to the $140 M_{\odot}$ "pP" model.

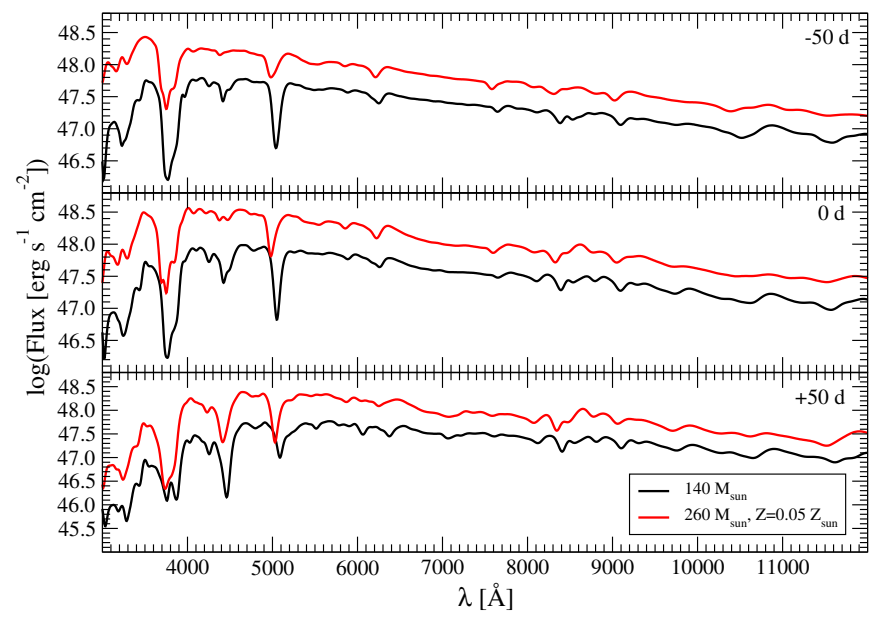

Figure 18. Comparison of the $140 M_{\odot}$ and the $0.05 Z_{\odot} 260 M_{\odot}$ model spectra at the contemporaneous epochs of -50 days (upper panel), 0 days (middle panel), and +50 days (lower panel) with respect to peak luminosity. There are no significant differences induced by different ZAMS metallicity in the spectral evolution of otherwise similar PISNe.

ure 17 shows the color evolution for these higher metallicity models.

The peak luminosity spectra for rotating PISN evolved with different metallicities at ZAMS (solid black, blue, and green curves in the right pane of Figure 16) all appear to be very similar with no distinguishable differences in any particular spectral features. This seems to be the case independently of phase as we illustrate in Figure 18, where the rotating zerometallicity $140 M_{\odot}$ model spectra are compared with those of the $0.05 Z_{\odot} 260 M_{\odot}$ model at the same phases (before, during, and after peak luminosity).

In contrast, for the $140 M_{\odot}$ models for which a metal content corresponding to $0.1 Z_{\odot}$ was added prior to import to $F L A S H$ ("pF" model; dashed red curve) and PHOENIX ("pP" model; solid red curve) differences are seen in the near-UV spectral flux and $\mathrm{Ca} H \& \mathrm{~K}$ absorption. For details on these models refer back to the discussion in Section 2.1. More specifically, the near-UV flux is suppressed in the " $\mathrm{pP}$ " model as compared to the original $140 M_{\odot}$ model. The "pF" model also shows a much smaller 
far UV flux suppression at $\lambda<4500 \AA$ while for the most part the spectrum is identical to the original $140 M_{\odot}$ model. The flux deficit is more dramatic in the "pP" case where the metallicity was added prior to calculating the PHOENIX spectra. This is because once the PISN explosion occur, the bulk of the additional metal content in the core of the progenitor contributes to the formation of ${ }^{56} \mathrm{Ni}$ and other iron-group elements through nuclear burning. By the time the SN photosphere expands, the blast profile is very similar to that of the $140 M_{\odot}$ model. In other words, the progenitor metallicity information is undecipherable in the post-explosion PISN spectra.

This is an important point of contrast with the results of (Kasen et al. 2011) illustrated in their Figure 11. They, too, find that increased metallicity has the effect to reduce the UV flux for otherwise identical PISN progenitors, but they also add the metal content immediately prior to doing the SEDONA radiative transfer calculations. Self-consistent evolution of PISNe with higher metal content from the ZAMS or even addition of extra metal abundances to a model prior to the explosion does not lead to significant differences in the spectra of our rotating models as compared to zero- or low-metallicity models. Note that, as we discuss in Section 4.3, our PISN models compare well with the (Kasen et al. 2011) models of the same ZAMS metallicity.

The radiative properties of PISNe appear to be degenerate across differences in ZAMS rotation rate and metallicity and seem to depend more on basic SN blast profile properties such as the temperature and velocity of the SN ejecta. Once the explosion sets in, the products of nuclear burning are predominantly IMEs in the outer- and iron-peak elements in the inner ejecta. Even the detailed spectral evolution of PISNe appears to be quite similar for models of different initial conditions. Pre-SN surface composition, on the other hand, seems to be the only factor leading to robust differences in the PISN spectra, as showcased by the $90 M_{\odot}$ model discussed above. Differences in pre-SN composition are primarily attributable to the extent of mass-loss suffered by the progenitor. As such, H/He-poor PISNe can be distinguished by the presence of $\mathrm{O}_{\mathrm{I}}$ absorption features in the optical, and that only if the appropriate temperature conditions are maintained in the $\mathrm{SN}$ ejecta.

\subsection{Comparison with other PISN Studies}

The LCs and color evolution we find for rotating PISNe have similar broad, qualitative properties with the non-LTE LCs of the non-rotating models studied by Kasen et al. (2011) and Dessart et al. (2013). To compare with these studies, we use our brightest zero-metallicity PISN model $\left(140 M_{\odot}\right)$. This model is the closest to the bare "helium" star He 100 models of both Kasen et al. (2011; He100K) and Dessart et al. (2013; He100D) in terms of $M_{\mathrm{CO}}, M_{\mathrm{Ni}}$ and final structural properties modulo differences in the surface abundances of $\mathrm{C}, \mathrm{N}$, and $\mathrm{O}$ that are enhanced in our model due to the effects of rotationally induced mixing.

The bolometric LC of our $140 M_{\odot}$ model is very close in terms of both peak luminosity $\left(M_{\text {bol,peak }}=-20.2 \mathrm{mag}\right.$, $\left.L_{\text {peak }} \simeq 4 \times 10^{43} \mathrm{erg}\right)$ and rise time to maximum $\left(t_{\text {peak }} \simeq\right.$ 170 days) with the He100K and He100D models. The same holds for the broadband LCs: the same behavior is seen in all three studies going from the bluer $(U)$ to the redder $(K)$ band. An exception is the range of peak filter magnitudes where our model is more in agreement with $\mathrm{He} 100 \mathrm{D}$ than with $\mathrm{He} 100 \mathrm{~K}$ who find a larger range $\left(\sim-19<M<-26\right.$ from $U_{\text {peak }}$ to $\left.K_{\text {peak }}\right)$. The peak absolute $V$ magnitudes recovered from all three studies are in good agreement $\left(M_{V} \sim-20.5\right.$ to -21$)$ regarding the differences in the initial progenitors. The color evolution of the

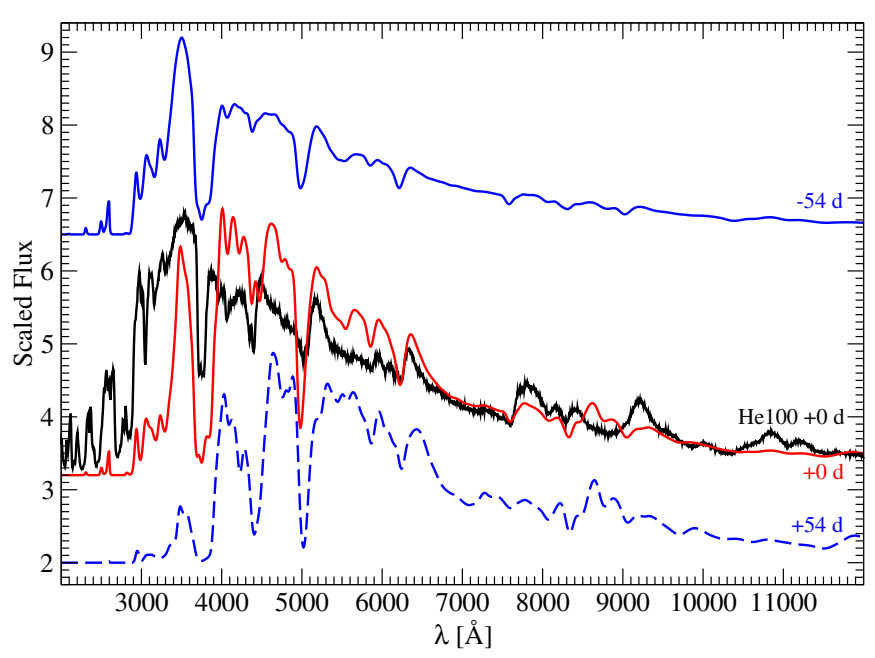

Figure 19. Comparison of the Kasen et al. (2011) He100 peak luminosity spectrum (black curve) with the -54 days, +54 days (solid and dashed blue curve, respectively), and peak luminosity (red curve) spectra of the $140 M_{\odot}$ model.

$140 M_{\odot}$ model is qualitatively similar to that of the He100D model, although we find it to be consistently redder for the rotating PISN. This could be due to differences between our LTE treatment and the Dessart et al. (2013) non-LTE approach.

A comparison of the spectral evolution between the three studies reveals several similarities as well. In terms of the peak luminosity spectrum, the strongest features seem to be recovered by all three approaches, somewhat resembling Type Ic SN spectra. More specifically the strong $\mathrm{Mg}$ I, Mg II, Si II, $\mathrm{Ca}$ II, and O I lines are recovered by all studies. Figure 19 shows a comparison of the $140 M_{\odot}$ spectra for different phases with respect to peak luminosity and the $\mathrm{He} 100 \mathrm{~K}$ peak spectrum.

Given the intrinsic differences of the progenitors, the agreement between the spectra of the $140 M_{\odot}$ model and He100K is very good in the optical (Figure 19). Obvious differences do exist in some near-IR features $(\lambda>8300 \AA)$ and the near-UV flux. We wish to note that besides examining the radiative properties of PISN this exercise also serves as direct comparison of model PISN spectra obtained from different codes. It is remarkable that given differences in the numerical treatment of radiative transfer between PHOENIX and SEDONA (which uses a Monte Carlo method) the main PISN spectroscopic characteristics recovered are in good agreement.

\subsection{Comparison with Observations}

Given the main properties of model PISN spectra that we have discussed in this work, it remains to examine if such events have been actually observed. At first glance, the general appearance of model PISN spectra appears to qualitatively resemble Type Ia and some normal Type Ic SN events with the lack of apparent $\mathrm{H}$ and He features and a strong presence of metals such as $\mathrm{O}, \mathrm{Mg}$, $\mathrm{Si}$, and $\mathrm{Ca}$. We attempt to illustrate these similarities in Figure 20 where we plot the peak luminosity $140 M_{\odot}$ and $0.1 Z_{\odot} 300 M_{\odot}$ spectra against near-maximum spectra of SN 2008D (Type Ib), SN 2011 fe (Type Ia), and SN 1994I (Type Ic). Obviously, SN 2008D is a bad match (also given the absence of He in our model spectra) but the other two events exhibit some similarities with model $0.1 Z_{\odot} 300 M_{\odot}$.

With regard to the proposed association between PISNe and a few observed SLSN events (Gal-Yam et al. 2009), we find that the emission properties of PISNe are quite different from 


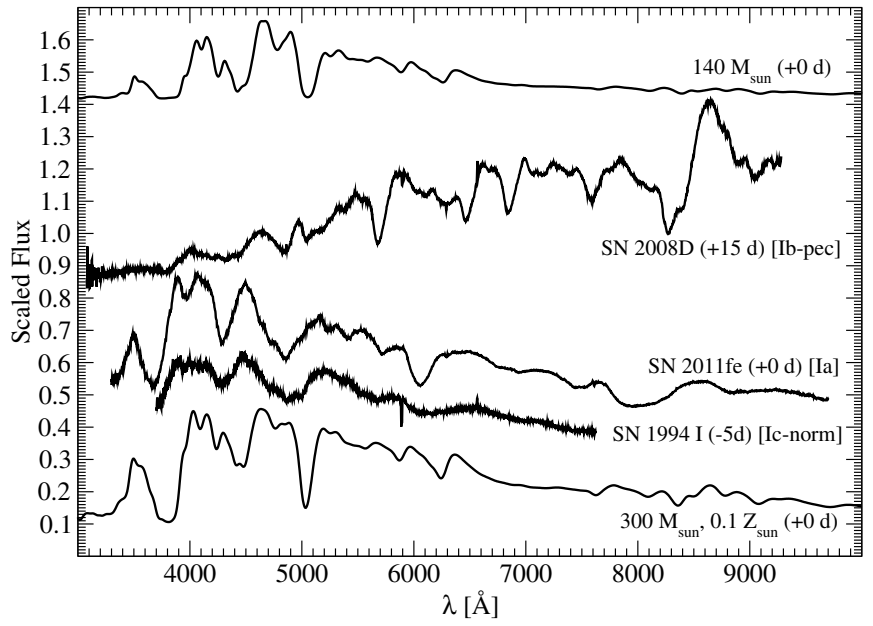

Figure 20. Comparison of the $140 M_{\odot}$ and the $300 M_{\odot}\left(Z=0.1 Z_{\odot}\right)$ rotating PISN model spectra at peak luminosity with observed spectra of the normal Type Ic SN 1994I (Filippenko et al. 1995), the Type Ia SN $2011 \mathrm{fe}$ (Nugent et al. 2011) and the peculiar Type Ib SN 2008D (Modjaz et al. 2009). The parentheses indicate the phase after peak luminosity in days and the brackets the SN type. All the observed SN spectra were downloaded from the Weizmann Interactive Supernova Data Repository (WISeREP; Yaron \& Gal-Yam 2012).

those of the proposed candidate SN 2007bi, in agreement with the results of Dessart et al. (2013). In particular, we find that PISNe of several metallicities and rotation rates are intrinsically red events and, in most cases, do not produce superluminous events due to the severe mass-loss suffered during the progenitor evolution. This is due to the fact that PISNe tend to have lower $M_{\mathrm{Ni}} / M_{\mathrm{f}}$ ratios than Type Ia or Type Ic-norm events leading to slowly evolving LCs with peak luminosities that span a large range from sub-luminous to some superluminous events in the most extreme cases. For very high ZAMS mass (>200 $M_{\odot}$ ), several solar masses of ${ }^{56} \mathrm{Ni}$ are produced powering a superluminous explosion. From the suite of models considered in our work with metallicities in agreement with that of the host of SN 2007bi, the only two that reach peak luminosities comparable to those of the event are the $0.1 Z_{\odot} 300 M_{\odot}$ and the $0.05 Z_{\odot} 260 M_{\odot}$ models. The observed $R$-band LC of SN 2007bi is compared against the LCs of these two models in Figure 21.

Based only on the LC, it can be deduced that a rotating PISN with $Z \simeq 0.05-0.1 Z_{\odot}$ is indeed a good model for SN 2007bi; however, a closer look at the spectral and color evolution of this event as compared with the model predictions reveals certain inconsistencies. The most important disagreement stems from a careful comparison of the model PISN spectra with those of SN 2007bi at contemporaneous epochs. This is an issue originally raised by Dessart et al. (2013) with regard to the apparent spectral agreement found between model He100K and the observed +54 days post-maximum spectrum of SN 2007bi by Kasen et al. (2011). The agreement was due to the fact that the He100K spectrum compared with the data was one at 50 days before peak, at an epoch when the PISN was still hot and blue. At later, post-maximum epochs, when the spectra of SN 2007bi were obtained, the model PISN spectra are much redder and strongly line-blanketed. A caveat to performing nearly contemporaneous model to data spectral comparisons is the fact that for many SLSN events, including SN 2007bi, the exact explosion date and therefore the phase of the observed spectrum are quite uncertain, as noted by Chatzopoulos et al. (2013b). Nevertheless, to be consistent with the other authors,

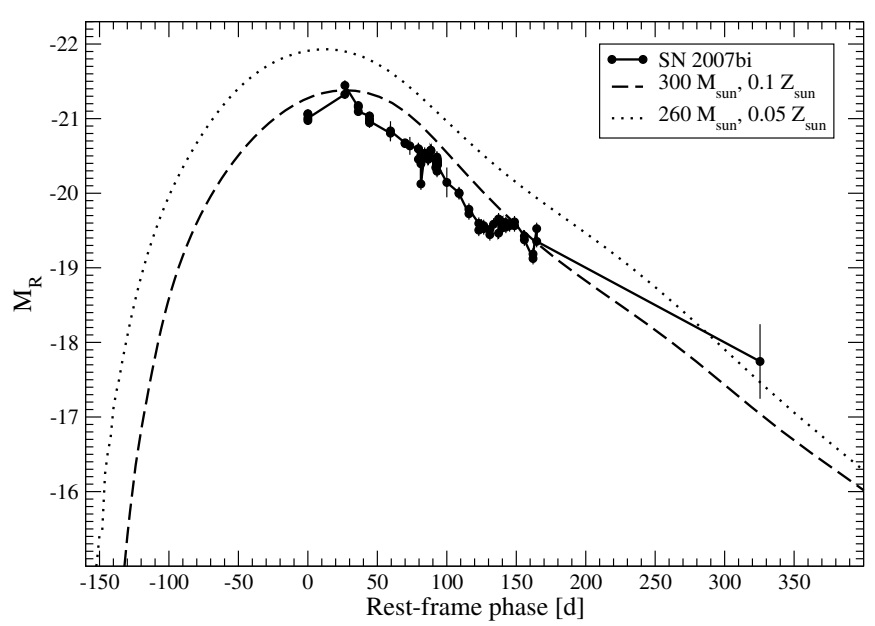

Figure 21. Observed $R$-band LC of SN 2007bi (Gal-Yam et al. 2009; solid curve) compared to the $R$-band model LCs of the rotating $0.1 Z_{\odot} 300 M_{\odot}$ (dashed curve) and $0.05 Z_{\odot} 260 M_{\odot}$ (dotted curve) models. Although PISN model LCs seem to be a good match to the observed LCs of some SLSNe, such as SN 2007bi, the predicted spectral evolution is remarkably different than the one observed.
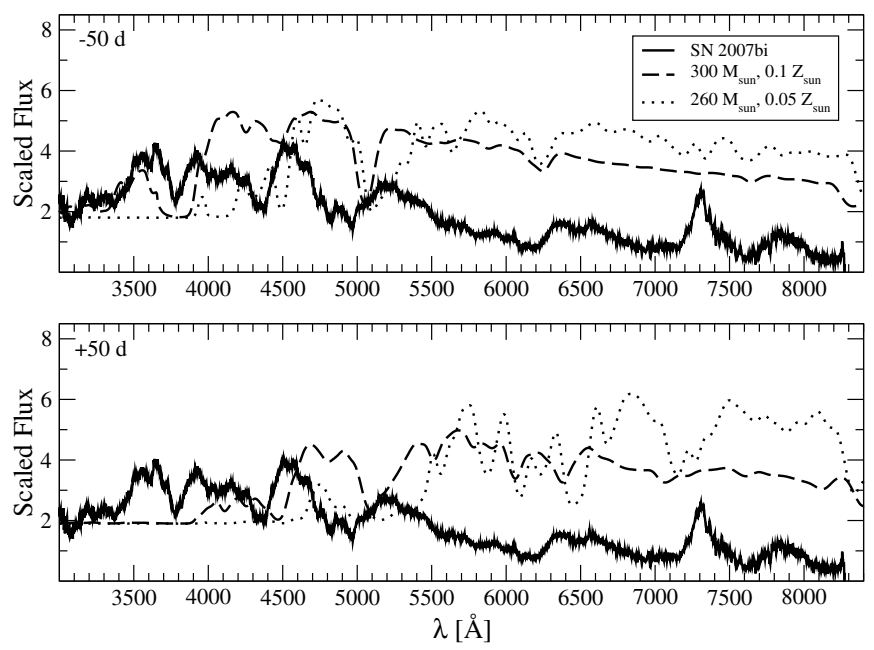

Figure 22. Comparison of the spectrum of SN 2007bi at $+54 \mathrm{~d}$ (Gal-Yam et al 2009 ; solid curve) with that of the $0.1 Z_{\odot} 300 M_{\odot}$ (dashed curve) and the $0.05 Z_{\odot} 260 M_{\odot}$ (dotted curve) model at -50 days (upper panel) and +50 days (lower panel) with respect to peak luminosity.

we will also accept the phase of the earliest observed SN 2007bi spectrum to be +54 days after peak luminosity.

Figure 22 shows the -50 days and +50 days (with regards to peak luminosity) spectra of the rotating $0.05 Z_{\odot} 260 M_{\odot}$ and $0.1 Z_{\odot} 300 M_{\odot}$ models compared with the observed spectrum of SN 2007bi +54 days after peak luminosity.

The comparison at the nearly contemporaneous epoch of +50 days (lower panel of Figure 22) reveals that the PISN models possess continua that are much redder and with different spectral features than the observations of SN 2007bi suggest. The agreement between the earlier model spectrum of the $0.1 Z_{\odot}$ $300 M_{\odot}$ model ( -50 days) and the data is slightly better but yet not nearly strong enough to suggest a physically consistent connection to the PISN mechanism. The color evolution of the model PISN spectra further affirms the fact that SN 2007bi was not a PISN. SN 2007bi retained blue color and a hot continuum for a long time after peak suggesting that a different, more efficient SN ejecta heating mechanism was at play and for a longer timescale than the radioactive decay of ${ }^{56} \mathrm{Ni}$. Potential 
alternative candidates are magneto-rotational energy injection due to the spin-down of a young magnetar (Dessart et al. 2012) and H-poor SN ejecta-CSM interaction (Chatzopoulos \& Wheeler 2012b; Chatzopoulos et al. 2013b). More detailed radiation hydrodynamics and non-LTE radiative transfer modeling is required to clearly identify the power-input mechanism for SN 2007bi and other H-poor SLSN (SLSN-I).

\section{DISCUSSION AND CONCLUSIONS}

In this paper we explored the radiative emission properties of model PISNe for a variety of progenitors of different ZAMS mass $\left(90-300 M_{\odot}\right)$, metallicity $\left(0-0.1 Z_{\odot}\right)$ and rotation rate. The analysis we performed was done in three basic stages: stellar evolution from ZAMS up to pre-SN with MESA, nuclear burning, and hydrodynamic evolution of the explosion phase up to post-SN shock break out with FLASH and radiative transfer with PHOENIX yielding the final model spectra and LCs. For a subset of our models (the zero-metallicity rotating 90-140 $M_{\odot}$ models) an additional step included the mapping of the pre-SBO profiles to the radiation hydrodynamics code $R A G E$ where the phase of SN shock breakout and the corresponding LCs and SEDs were computed.

As has been discussed in past studies (Chatzopoulos \& Wheeler 2012a; Yoon et al. 2012) the main effects of rotation on the evolution of PISN progenitors is the enhanced mixing leading to the formation of larger $\mathrm{CO}$ cores for initially smaller ZAMS mass as compared to the case of zero rotation, and the increased mass-loss suffered leading to bare CO stars with little or no $\mathrm{He}$ in their envelopes prior to explosion. As such, the pre-explosion zero-metallicity models we consider here were compact $\left(R_{\mathrm{f}}<2 \times 10^{11} \mathrm{~cm}\right)$ and dense all the way to the surface. The RAGE simulations revealed that the SBO SEDs of these events are significantly hotter than those implied for RSG and BSG progenitors (Kasen et al. 2011) peaking in the hard X-rays (1-100 keV). Nevertheless, the combination of lower pre-SN radii, higher $\mathrm{SN}$ ejecta temperatures and velocities and harder emission leads to SBO bolometric LCs with peaks similar to those found for RSG and BSG progenitors $\left(10^{45}-10^{46}\right.$ erg s$\left.^{-1}\right)$.

The main, long-duration part of the LCs powered by the radioactive decays of ${ }^{56} \mathrm{Ni}$ and ${ }^{56} \mathrm{Co}$ was studied for all models using the PHOENIX results. For most PISN models, the implied $M_{\mathrm{Ni}} / M_{\mathrm{ej}}$ ratios were below $10 \%$, a value close to Type IIP events leading to intrinsically red colors, as noted by Dessart et al. (2012). As such, most PISN exhibited peak luminosities spanning the range observed for normal Type II and Ib/c events. superluminous LCs were found only in the most massive non-zero-metallicity $260 M_{\odot}$ and $300 M_{\odot}$ models. We stress that superluminous PISN LCs are possible for zero-metallicity models with higher masses than the ones we studied in this work (>140 $M_{\odot}$ ). Nevertheless, all models evolved with redder colors than those observed for SLSN-I and, more specifically, SN 2007bi.

We investigated the potential to distinguish rotation and metallicity information from the observed PISN spectra and came to the conclusion that the spectroscopic properties of these events are quite degenerate and any attempt to uncover them based on observed spectra would be highly uncertain. More specifically, we find that the increased abundances of $\mathrm{C}$, $\mathrm{N}$, and $\mathrm{O}$ in the outer layers of the rotating models do not have a significant effect in the spectra and that once the explosion setsin the products of nuclear burning are the same regardless of the
pre-SN rotational mixing and metallicity. The peak luminosity spectra, but also the spectral evolution of PISN in general, appear to be quite similar across different initial metallicity and rotation rate. This is in contradiction with the result of Kasen et al. (2011), which increased metallicity leads to suppressed near-UV flux and differences in some spectral features. This difference is attributable to the fact that in their approach the extra metals were added after the PISN explosion and prior to the free expansion phase where the radiative transfer calculation is done. Self-consistent evolution of PISN with ZAMS metallicity results in final spectra with similar properties.

Most of the spectroscopic properties across the PISN parameter space arise due to fundamental differences in pre-SN composition and different SN ejecta masses, temperatures, and velocities. The more massive models have a somewhat bluer continuum with fewer lines in the optical than then less massive ones. These points are well illustrated by our $90 M_{\odot}$ model that was a pure oxygen star prior to explosion. The oxygen-rich composition together with the high SN ejecta temperature leads to spectra with strong $\mathrm{O}_{\mathrm{I}}$ absorption features that are distinguishable from those of the other models.

Our model PISN spectra compare well against those calculated by Kasen et al. (2011) and Dessart et al. (2013) for similar progenitors (more specifically their bare $100 M_{\odot}$ He core model He100). This confirms that regardless of the differences in numerical methods between different codes and LTE versus nonLTE treatment the results for the main emission characteristics of PISN are consistent.

Although the $R$-band LC of the $0.1 Z_{\odot} 300 M_{\odot}$ model reproduces the main features of the LC of SN 2007bi, it is not sufficient information to categorize the event as a PISN. Comparison of our model spectra against the observed +54 days spectrum of the superluminous SN 2007bi at contemporaneous epochs confirms that SN 2007bi was not a PISN, in agreement with the result of Dessart et al. (2013). More specifically, PISNe appear to have redder color evolution and different spectroscopic features compared to all observed SLSNe. This could be due to the fact that most SLSNe have been observed in environments with metallicities $\sim 0.45 Z_{\odot}$ (Lunnan et al. 2014), disfavoring the formation of very massive pre-SN CO cores due to strong mass-loss. This value is near the upper limit metallicity of $1 / 3 Z_{\odot}$ for the most massive progenitors assuming standard initial mass functions (IMF; Langer et al. 2007). In addition, other models of PISN present in the literature with LCs in agreement with that of SN 2007bi have adopted a progenitor metallicity that is far lower than that of the host galaxy of the event (Kozyreva et al. 2014a) or have made simplifying assumptions with regards to the progenitor mass-loss history (Kasen et al. 2008; Gal-Yam et al. 2009). Given these results, we conclude that all SLSN events observed so far must be powered via alternative mechanisms with the most promising candidates being radiation from a magnetar spin-down (Dessart et al. 2012; McCrum et al. 2014) and CSM interaction of the H-rich and H-poor variety (Chatzopoulos \& Wheeler 2012b; Chatzopoulos et al. 2013b; Benetti et al. 2014; Nicholl et al. 2014).

The PISN phenomenon is expected to be more prevalent in ultra-low- and zero-metallicity environments appropriate to the conditions of the early universe. Some Population III star-formation simulations predict the first stars to be massive and rotating (Stacy et al. 2012, 2013), well within the PISN parameter space. As such, the future of observational research in the subject seems to rest on the shoulders of exciting upcoming missions such as WFIRST and JWST. 
We thank Wesley Even, Sean M. Couch, Donald Q. Lamb, Ryan Chornock, Ken Chen, Alexandra Kozyreva, and Ragnhild Lunnan for useful discussions and comments. This work was supported in part by the STScI grants AR 12820 and 13276 and the National Science Foundation under grants AST-0909132 and PHY-0822648 for the Physics Frontier Center "Joint Institute for Nuclear Astrophysics" (JINA). E.C. thanks the Enrico Fermi Institute for its support via the Enrico Fermi Fellowship.

\section{REFERENCES}

Abel, T., Anninos, P., Norman, M. L., \& Zhang, Y. 1998, ApJ, 508, 518 Barkat, Z., Rakavy, G., \& Sack, N. 1967, PhRvL, 18, 379

Benetti, S., Nicholl, M., Cappellaro, E., et al. 2014, MNRAS, 441, 289

Bromm, V., Coppi, P. S., \& Larson, R. B. 2002, ApJ, 564, 23

Bromm, V., \& Larson, R. B. 2004, ARA\&A, 42, 79

Bromm, V., Yoshida, N., Hernquist, L., \& McKee, C. F. 2009, Natur, 459, 49

Brott, I., de Mink, S. E., Cantiello, M., et al. 2011a, A\&A, 530, A115

Brott, I., Evans, C. J., Hunter, I., et al. 2011b, A\&A, 530, A116

Chatzopoulos, E., \& Wheeler, J. C. 2012a, ApJ, 748, 42

Chatzopoulos, E., \& Wheeler, J. C. 2012b, ApJ, 760, 154

Chatzopoulos, E., Wheeler, J. C., \& Couch, S. M. 2013a, ApJ, 776, 129

Chatzopoulos, E., Wheeler, J. C., Vinko, J., Horvath, Z. L., \& Nagy, A. 2013b, ApJ, 773, 76

Chatzopoulos, E., Wheeler, J. C., Vinko, J., et al. 2011, ApJ, 729, 143

Chen, K.-J., Heger, A., Woosley, S., Almgren, A., \& Whalen, D. 2014a, ApJ, 792, 44

Chen, K.-J., Woosley, S., Heger, A., Almgren, A., \& Whalen, D. 2014b, ApJ, 792,28

Crowther, P. A., Schnurr, O., Hirschi, R., et al. 2010, MNRAS, 408, 731

de Souza, R. S., Ishida, E. E. O., Whalen, D. J., Johnson, J. L., \& Ferrara, A. 2014, MNRAS, 442, 1640

Dessart, L., Hillier, D. J., Waldman, R., Livne, E., \& Blondin, S. 2012, MNRAS, 426, L76

Dessart, L., Waldman, R., Livne, E., Hillier, D. J., \& Blondin, S. 2013, MNRAS, 428, 3227

Dubey, A., Reid, L. B., Weide, K., et al. 2009, arXiv:0903.4875

Dufton, P. L., Dunstall, P. R., Evans, C. J., et al. 2011, ApJL, 743, L22

Ekström, S., Georgy, C., Eggenberger, P., et al. 2012, A\&A, 537, A146

Ekström, S., Meynet, G., Chiappini, C., Hirschi, R., \& Maeder, A. 2008, A\&A, 489,685

Filippenko, A. V., Barth, A. J., Matheson, T., et al. 1995, ApJL, 450, L11

Frey, L. H., Even, W., Whalen, D. J., et al. 2013, ApJS, 204, 16

Fryxell, B., Olson, K., Ricker, P., et al. 2000, ApJS, 131, 273

Gal-Yam, A. 2012, Sci, 337, 927

Gal-Yam, A., Mazzali, P., Ofek, E. O., et al. 2009, Natur, 462, 624

Gittings, M., Weaver, R., Clover, M., et al. 2008, CS\&D, 1, 015005

Greif, T. H., Springel, V., White, S. D. M., et al. 2011, ApJ, 737, 75

Hachinger, S., Mazzali, P. A., Sullivan, M., et al. 2013, MNRAS, 429, 2228

Hauschildt, P. H. 1992, JQSRT, 47, 433

Hauschildt, P. H., \& Baron, E. 1999, JCoAM, 109, 41
Hauschildt, P. H., \& Baron, E. 2004, A\&A, 417, 317

Heger, A., Langer, N., \& Woosley, S. E. 2000, ApJ, 528, 368

Heger, A., \& Woosley, S. E. 2002, ApJ, 567, 532

Hillier, D. J., \& Dessart, L. 2012, MNRAS, 424, 252

Hummel, J. A., Pawlik, A. H., Milosavljević, M., \& Bromm, V. 2012, ApJ, 755,72

Joggerst, C. C., \& Whalen, D. J. 2011, ApJ, 728, 129

Kasen, D., \& Bildsten, L. 2010, ApJ, 717, 245

Kasen, D., Heger, A., \& Woosley, S. 2008, in AIP Conf. Proc. 990, First Stars III, ed. B. W. O'Shea \& A. Heger (Melville, NY: AIP), 263

Kasen, D., Woosley, S. E., \& Heger, A. 2011, ApJ, 734, 102

Kozyreva, A., Blinnikov, S., Langer, N., \& Yoon, S.-C. 2014a, A\&A, 565, A70

Kozyreva, A., Yoon, S.-C., \& Langer, N. 2014b, A\&A, 566, A146

Langer, N., Norman, C. A., de Koter, A., et al. 2007, A\&A, 475, L19

Lucy, L. B. 1991, ApJ, 383, 308

Lunnan, R., Chornock, R., Berger, E., et al. 2014, ApJ, 787, 138

Maeder, A., \& Meynet, G. 2011, arXiv:1109.6171

McCrum, M., Smartt, S. J., Kotak, R., et al. 2014, MNRAS, 437, 656

Modjaz, M., Li, W., Butler, N., et al. 2009, ApJ, 702, 226

Moriya, T. J., Blinnikov, S. I., Tominaga, N., et al. 2013, MNRAS, 428,1020

Moriya, T. J., \& Tominaga, N. 2012, ApJ, 747, 118

Nicholl, M., Smartt, S. J., Jerkstrand, A., et al. 2014, MNRAS, 444, 2096

Nugent, P. E., Sullivan, M., Cenko, S. B., et al. 2011, Natur, 480, 344

Ober, W. W., El Eid, M. F., \& Fricke, K. J. 1983, A\&A, 119, 61

Olson, G. L., \& Kunasz, P. B. 1987, JQSRT, 38, 325

Pan, T., Kasen, D., \& Loeb, A. 2012, MNRAS, 422, 2701

Paxton, B., Bildsten, L., Dotter, A., et al. 2011, ApJS, 192, 3

Paxton, B., Cantiello, M., Arras, P., et al. 2013, ApJS, 208, 4

Quimby, R. M., Aldering, G., Wheeler, J. C., et al. 2007, ApJL, 668, L99

Rakavy, G., \& Shaviv, G. 1967, ApJ, 148, 803

Rakavy, G., Shaviv, G., \& Zinamon, Z. 1967, ApJ, 150, 131

Scannapieco, E., Madau, P., Woosley, S., Heger, A., \& Ferrara, A. 2005, ApJ, 633,1031

Smith, N., Li, W., Foley, R. J., et al. 2007, ApJ, 666, 1116

Smith, N., \& McCray, R. 2007, ApJL, 671, L17

Spruit, H. C. 1999, A\&A, 349, 189

Spruit, H. C. 2002, A\&A, 381, 923

Stacy, A., Greif, T. H., \& Bromm, V. 2012, MNRAS, 422, 290

Stacy, A., Greif, T. H., Klessen, R. S., Bromm, V., \& Loeb, A. 2013, MNRAS, 431,1470

Stoll, R., Prieto, J. L., Stanek, K. Z., \& Pogge, R. W. 2013, ApJ, 773, 12

van Rossum, D. R. 2012a, ApJ, 756, 31

van Rossum, D. R. 2012b, arXiv:1208.3781

Whalen, D. J. 2013, AcPol, 53, 573

Whalen, D. J., Even, W., Frey, L. H., et al. 2013a, ApJ, 777, 110

Whalen, D. J., Fryer, C. L., Holz, D. E., et al. 2013b, ApJL, 762, L6

Woosley, S. E. 2010, ApJL, 719, L204

Woosley, S. E., Blinnikov, S., \& Heger, A. 2007, Natur, 450, 390

Yaron, O., \& Gal-Yam, A. 2012, PASP, 124, 668

Yoon, S.-C., Dierks, A., \& Langer, N. 2012, A\&A, 542, A113

Yoon, S.-C., \& Langer, N. 2005, A\&A, 443, 643

Yusof, N., Hirschi, R., Meynet, G., et al. 2013, MNRAS, 433, 1114 